\title{
On a periodic dividend barrier strategy in the dual model with continuous monitoring of solvency
}

\author{
Benjamin Avanzi ${ }^{\mathrm{a}}$, Eric C.K. Cheung, ${ }^{*}$, Bernard Wong $^{\mathrm{a}}$, Jae-Kyung Woo $^{\mathrm{b}}$ \\ ${ }^{a}$ School of Risk and Actuarial Studies, Australian School of Business \\ University of New South Wales Sydney, NSW 2052, Australia \\ ${ }^{b}$ Department of Statistics and Actuarial Science \\ University of Hong Kong, Pokfulam, Hong Kong
}

\begin{abstract}
We consider the dual model, which is appropriate for modelling the surplus of companies with deterministic expenses and stochastic gains, such as pharmaceutical, petroleum or commission-based companies. Dividend strategies for this model that can be found in the literature include the barrier strategy (e.g., Avanzi et al., 2007) and the threshold strategy (e.g., Cheung, 2008), where dividend decisions are made continuously. While in practice the financial position of a company is typically monitored frequently, dividend decisions are only made periodically along with the publication of its books. In this paper, we introduce a dividend barrier strategy whereby dividend decisions are made only periodically, but still allow ruin to occur at any time (as soon as the surplus is exhausted). This is in contrast to Albrecher et al. (2011a), who introduced periodic dividend payments in the Cramér-Lundberg surplus model, albeit with periodic ruin opportunities as well.

Under the assumption that the time intervals between dividend decisions are Erlang $(n)$ distributed, we derive integro-differential equations for the Laplace transform of the time to ruin and the expected present value of dividends until ruin. These are then solved with the help of probabilistic arguments. We also provide a recursive algorithm to compute these quantities. Finally, some numerical studies are presented, which aim at illustrating how our assumptions about dividend payments and ruin occurrence compare with those of the classical barrier strategy.
\end{abstract}

Key words: Dual model, Barrier strategy, Erlangization, Dividends, Ruin.

JEL codes: C44, C61, G24, G32, G35

\section{Introduction}

\subsection{Motivation and background}

In actuarial risk theory, stochastic processes are developed to model the surplus of risk businesses in light of the stability problem (Bühlmann, 1970). Several stability criteria are typically considered, including the probability of ruin (Lundberg, 1909; Cramér, 1955) and dividends (de Finetti, 1957). In its modified formulation, Bruno de Finetti (1957) introduced dividends to make the dynamic behaviour of the surplus process more realistic (see also Avanzi, 2009, for a discussion and a review of the subsequent literature). Usually, the modelling approach is to allow dividends to be paid at any time, depending on the level of the surplus. In this paper, we consider a dividend strategy whereby dividend decisions are made only periodically, which is arguably more realistic. On the other hand, while in practice the books of a company are only

\footnotetext{
*Corresponding author. Tel.: +852 2857 8315; fax: +852 28589041.

Email addresses: b.avanzi@unsw.edu.au (Benjamin Avanzi), eckc@hku.hk (Eric C.K. Cheung), bernard.wong@unsw.edu.au (Bernard Wong), jkwoo@hku.hk (Jae-Kyung Woo)
} 
published periodically along with dividend announcements, its financial position is typically monitored more frequently. As a consequence, we will allow ruin to occur at any time, as soon as the surplus becomes negative.

We consider companies with deterministic expenses and stochastic gains, such as pharmaceutical, petroleum or commission-based companies. The surplus of such companies (before dividends) can be described as

$$
U(t)=u-c t+\sum_{i=1}^{N(t)} Y_{i}, \quad t \geq 0,
$$

where $u=U(0) \geq 0$ is the initial surplus, and $c>0$ is the constant rate of expenses per unit time. The total amount of gains follows a compound Poisson process with intensity $\lambda>0$ whose individual gains have a common probability density function $p(\cdot)$, Laplace transform $\widetilde{p}(\cdot)$ and mean $\mu=E\left[Y_{1}\right]$. The time of ruin of $\{U(t)\}_{t \geq 0}$ is defined by $\tau=\inf \{t \geq 0: U(t) \leq 0\}$. Furthermore, we assume that the business is profitable, i.e. $\lambda \mu>c$. When considering the dynamics of (1.1) it becomes apparent that ruin should be allowed to occur at any time. Indeed, when the surplus is exhausted the company will not be able to fund its continuous expenses anymore and will have to cease operations.

Because of its duality to the classical Cramér-Lundberg model, the model described in (1.1) was first referred to as the 'dual risk model' by Mazza and Rullière (2004). In addition to its afore-mentioned interpretation, Seal (1969, p.116) argued that the process (1.1) may also be suitable for annuity or pension funds, where the insurance company pays annuities and randomly earns a portion of the reserves when a policyholder dies.

Classical ruin probability results for model (1.1) can be found in e.g. Cramér (1955, Section 5.13), Takács (1967, pp.152-154), Seal (1969, pp.116-119) and Grandell (1991, p.8). A generalization is the dual Sparre Andersen or renewal risk model in which the inter-arrival times of the gains are not necessarily exponentially distributed. Cheung (2012) studied a Gerber-Shiu type function (see Gerber and Shiu, 1998) in such a model, whereas Mazza and Rullière (2004) showed that the time of ruin in the dual model can be related to that in the standard risk model. The dual Markov-modulated risk model in which dependence among gain sizes and their inter-arrival times is introduced via an underlying Markovian environment was also investigated by Zhu and Yang (2008). See also Cheung $(2010,2011)$ for the dual risk model with additional possibility of downward jumps.

Avanzi et al. (2007), Avanzi and Gerber (2008), Bayraktar and Egami (2008), Cheung and Drekic (2008), Gerber and Smith (2008) and Avanzi et al. (2011) considered barrier strategies in which any excess of the surplus process over a fixed level $b>0$ is immediately paid to shareholders as dividend until ruin occurs. Alternative dividend strategies include threshold-type strategy where dividend is paid a fixed rate whenever the surplus is above $b$ (Cheung, 2008; $\mathrm{Ng}, 2009,2010$ ). We also refer interested readers to Albrecher et al. (2008) for a dual model with tax payments.

Note that the idea of periodic dividends was first introduced by Albrecher et al. (2011a) in the CramérLundberg model, where both dividend payments and ruin can only happen periodically. Periodic dividends were also considered in a Brownian risk model by Albrecher et al. (2011b), where dividends can be paid at any time with probability $\gamma d t$ when the surplus is positive (i.e. inter-dividend-decision times are exponential), and where ruin can happen at any time with probability $\omega(x) d t$ when the surplus $x$ is negative. In contrast, we assume that ruin occurs immediately once the surplus becomes negative, and consider a different surplus model.

\subsection{The introduction of periodic dividends}

It follows from the results by Miyasawa (1962) and Takeuchi (1962) in a discrete setting that the classical barrier strategy should be the one that maximizes the expected present value of dividends until ruin in the dual model. This is yet to be shown in general, but some specific cases were considered (see Bayraktar and Egami, 2008; Avanzi et al., 2011). As explained above, we consider only periodic dividend payments. Formally, we assume that these can happen only at time points $\left\{Z_{k}\right\}_{k=1}^{\infty}$. The payout behaviour is then one of a barrier strategy, i.e. if at any time $Z_{k}$ the surplus is above a fixed level $b>0$, then the excess over $b$ 
is paid out as dividend. This is in contrast to the classical barrier strategy whereby any overshoot over $b$ is immediately paid out as dividend as decisions are made continuously.

The time between successive dividend decision time points is assumed to be $\operatorname{Erlang}(n)$ distributed. More specifically, we define

$$
Z_{k}=\sum_{i=1}^{k} T_{i}, \quad k=1,2, \ldots
$$

to be the $k$-th time that the decision of whether to pay a dividend to the shareholders is made, where $\left\{T_{i}\right\}_{i=1}^{\infty}$ is a sequence of independent and identically distributed (i.i.d.) $\operatorname{Erlang}(n) \operatorname{random}$ variables with scale parameter $\gamma>0$ (independent of $\{N(t)\}_{t \geq 0}$ and $\left.\left\{Y_{i}\right\}_{i=1}^{\infty}\right)$ possessing common density

$$
f_{T}(t)=\frac{\gamma^{n} t^{n-1} e^{-\gamma t}}{(n-1) !}, \quad t>0
$$

This choice is motivated by several factors. Firstly, it makes computation more tractable. Indeed, each random variable $T_{i}$ can be seen as the sum of $n$ i.i.d. exponential variables with mean $1 / \gamma$, which allows us to profit from their memoryless properties. This so-called 'Erlangization' technique was proposed by Asmussen et al. (2002), and its computational advantage has been exploited by e.g. Stanford et al. (2005, 2011), Ramaswami et al. (2008) and Landriault et al. (2012) in various finite-time ruin problems as well as Parisian-type ruin problems. Secondly, fixing the mean $E\left[T_{1}\right]=n / \gamma=h$ while increasing $n$ (and increasing $\gamma$ ) will approximate a fixed time horizon $h$ such that deterministic inter-dividend-decision times are obtained as a limit (see also Section 5.2).

When using this Erlangization technique, the surplus process $\left\{U_{b}(t)\right\}_{t \geq 0}$ (after distribution of dividends) needs to be defined in-between each Erlang time interval. To this end, we shall introduce the auxiliary processes $\left\{W_{k}(t)\right\}_{t \geq 0}$ for $k=1,2, \ldots$ The dynamics of $\left\{U_{b}(t)\right\}_{t \geq 0}$ and $\left\{W_{k}(t)\right\}_{t \geq 0}$ can be described recursively via the two coupled equations

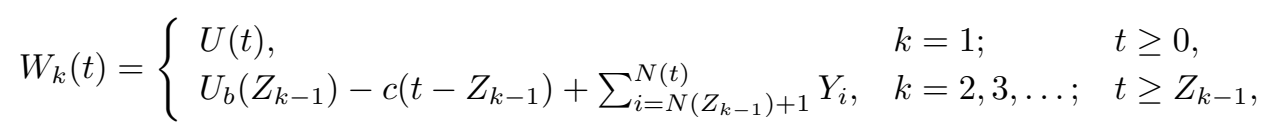

and for $k=1,2, \ldots$,

$$
U_{b}(t)= \begin{cases}W_{k}(t), & Z_{k-1}<t<Z_{k} \\ \min \left(W_{k}\left(Z_{k}\right), b\right), & t=Z_{k}\end{cases}
$$

Here we define $Z_{0}=0^{-}$and $U_{b}(0)=u$ even if $u>b$, i.e. time 0 is not assumed to be a dividend decision time (without loss of generality). by

The time of ruin in the present model is $\tau_{b}=\inf \left\{t \geq 0: U_{b}(t) \leq 0\right\}$, and its Laplace transform is defined

$$
\phi(u ; b)=E\left[e^{-\delta \tau_{b}} 1\left\{\tau_{b}<\infty\right\} \mid U_{b}(0)=u\right], \quad u \geq 0,
$$

where $1\{A\}$ represents the indicator function of the event $A$, and the Laplace transform argument $\delta \geq 0$ may also be interpreted as the force of interest. By defining $k_{b}=\max \left\{k \geq 0: Z_{k} \leq \tau_{b}\right\}$, then $k_{b}$ clearly represents the number of dividend decisions until ruin.

The expected discounted dividends payable until ruin is given by

$$
V(u ; b)=E\left[\sum_{k=1}^{k_{b}} e^{-\delta Z_{k}}\left[W_{k}\left(Z_{k}\right)-b\right]_{+} \mid U_{b}(0)=u\right], \quad u \geq 0,
$$

where $a_{+}=\max (a, 0)$. The functions $\phi(u ; b)$ and $V(u ; b)$ are the key quantities of interest in this paper. We remark that if instead one assumes that time 0 is a dividend decision time, then for $u>b$ one has $\phi(u ; b)=\phi(b ; b)$ and $V(u ; b)=u-b+V(b ; b)$ where $\phi(b ; b)$ and $V(b ; b)$ are obtainable from the current model with time 0 not being a dividend decision time. Please refer to Figure 1 for a typical sample path of the modified surplus process $\left\{U_{b}(t)\right\}_{t \geq 0}$. 


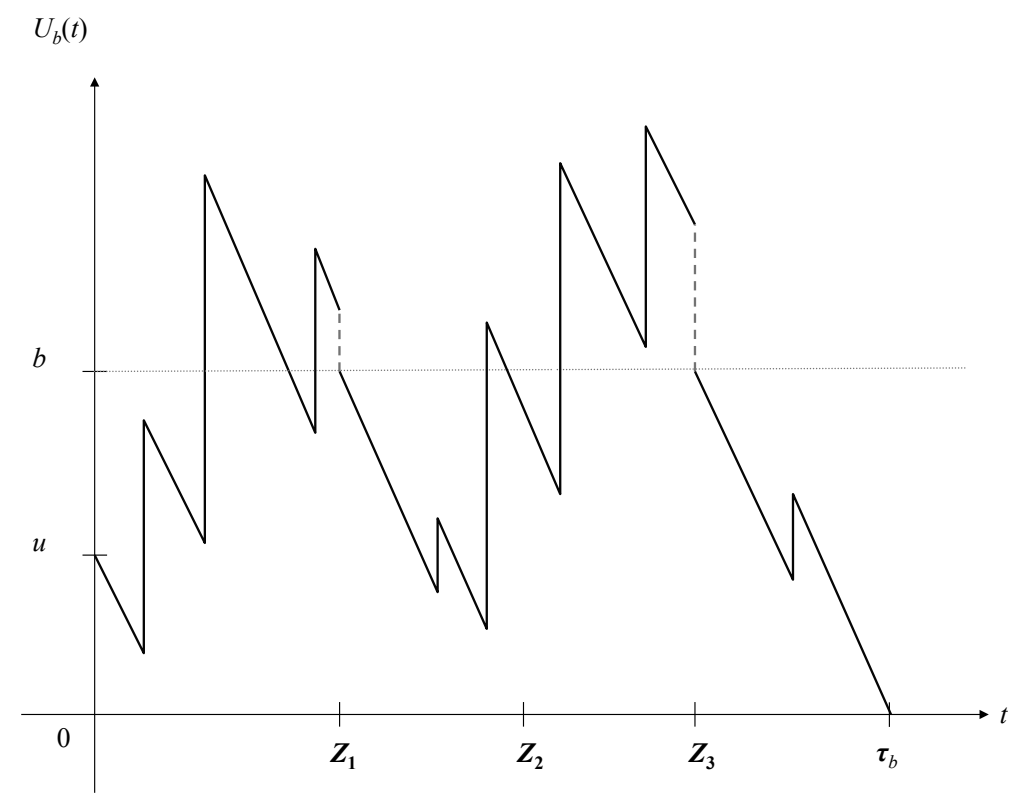

Figure 1: Typical sample path of the modified surplus process $\left\{U_{b}(t)\right\}_{t \geq 0}$

In our analysis, the notion of Laplace transforms will be used for various functions. The Laplace transform of a function $f(\cdot)$ on $(0, \infty)$ (which does not necessarily have to be a density) will be denoted by, for any complex number $s$ with $\operatorname{Re}(s) \geq 0$,

$$
\widetilde{f}(s)=\int_{0}^{\infty} e^{-s x} f(x) d x .
$$

\subsection{Structure of the paper}

This paper is organized as follows. In Section 2, the Laplace transform of the time of ruin $\phi(u ; b)$ is studied. Piecewise integro-differential equations are derived in Section 2.1 for $0<u<b$ and $u>b$ along with the appropriate boundary conditions. Although we are not able to solve the system directly, probabilistic arguments can be used to determine the solution form for $u>b$ apart from some unknown constants, and this will be the subject matter of Section 2.2. Applying the results of Section 2.2, the solution to $\phi(u ; b)$ is fully characterized in Sections 2.3 and 2.4. Section 3 is concerned with the dividend function $V(u ; b)$. Our approach there is similar to that of Section 2 (and we hence use a similar layout), but it requires considerable amount of extra effort to obtain the correct solution form. Section 4 studies the special case in which both the claim amounts and the inter-dividend-decision times are exponentially distributed. Explicit formulas for $\phi(u ; b)$ and $V(u ; b)$ are given, and the optimal dividend barrier is identified. In Section 5 , we present some numerical studies which aim at illustrating how our assumptions about dividend payments and ruin compare with that of the classical barrier strategy, as well as the one introduced in Albrecher et al. (2011a). We also shed some light on the behaviour of the (numerically) optimal barrier level in our model.

\section{Laplace transform of the time of ruin}

To take advantage of an underlying phase-type structure of the problem, in what follows we shall treat the $\operatorname{Erlang}(n)$ distribution (i.e. the generic inter-dividend-decision time) as the sum of $n$ i.i.d. exponential variables. For $i=1,2, \ldots, n$, we let $\phi_{i}(u ; b)$ be the Laplace transform of the time of ruin given that the surplus level is $u$ and the time remaining until the next dividend decision time is distributed as $\operatorname{Erlang}(n-i+1)$. 
Clearly, $\phi(u ; b) \equiv \phi_{1}(u ; b)$. The subscript $i$ in $\phi_{i}(u ; b)$ can be regarded as an index that keeps track of the 'Erlang clock'. Whenever an exponential variable is realized, the index $i$ will (1) increase by 1 if $i=1,2, \ldots, n-1$; or $(2)$ revert to $i=1$ if $i=n$ due to a dividend decision. Therefore, each exponential constituting the $\operatorname{Erlang}(n)$ inter-dividend-decision time is like a clock tick.

\subsection{Integro-differential equations}

For $u>0$, we start by considering all possible events over a very small time interval $[0, h]$ and noting that there is a competition between two independent Poisson processes with rates $\lambda$ and $\gamma$. When $i=$ $1,2, \ldots, n-1$, no dividend can be paid at time $h$, and we arrive at

$\phi_{i}(u ; b)=[1-(\lambda+\gamma) h] e^{-\delta h} \phi_{i}(u-c h ; b)+\gamma h e^{-\delta h} \phi_{i+1}(u-c h ; b)+\lambda h e^{-\delta h} \int_{0}^{\infty} \phi_{i}(u-c h+y ; b) p(y) d y+o(h)$.

On the other hand, when $i=n$, if (1) the Poisson process with rate $\gamma$ first has an event; and (2) the surplus is above level $b$ at the time of such an event, then dividend is paid thereby reducing the surplus level to $b$. This leads to

$$
\begin{aligned}
\phi_{n}(u ; b)= & {[1-(\lambda+\gamma) h] e^{-\delta h} \phi_{n}(u-c h ; b)+\gamma h e^{-\delta h}\left[\phi_{1}(u-c h ; b) 1\{u-c h \leq b\}+\phi_{1}(b ; b) 1\{u-c h>b\}\right] } \\
& +\lambda h e^{-\delta h} \int_{0}^{\infty} \phi_{n}(u-c h+y ; b) p(y) d y+o(h)
\end{aligned}
$$

By letting $h \rightarrow 0$ in (2.1) and (2.2), for each $i=1,2, \ldots, n$ one easily observes the left-continuity of $\phi_{i}(u ; b)$ for $u>0$. If one instead starts the above argument at an initial surplus level of $u+c h$, right-continuity can be established as well.

Since the function $\phi_{i}(u ; b)$ exhibits different behavior depending on whether $u$ is below or above $b$ (as evident from $(2.2)$ ), it is further defined in a piecewise manner as

$$
\phi_{i}(u ; b)= \begin{cases}\phi_{L, i}(u ; b), & 0 \leq u<b \\ \phi_{U, i}(u ; b), & u \geq b\end{cases}
$$

where ' $L$ ' and ' $U$ ' stand for 'Lower' and 'Upper' respectively. For later use we also define the row vectors $\underline{\phi}_{L}(u ; b)=\left(\phi_{L, 1}(u ; b), \phi_{L, 2}(u ; b), \ldots, \phi_{L, n}(u ; b)\right)^{T}$ and $\underline{\phi}_{U}(u ; b)=\left(\phi_{U, 1}(u ; b), \phi_{U, 2}(u ; b), \ldots, \phi_{U, n}(u ; b)\right)^{T}$, where the superscript ' $T$ ' denotes a transpose. The previously established continuity of $\phi_{i}(u ; b)$ for $u>0$ implies

$$
\phi_{L, i}(b ; b)=\phi_{U, i}(b ; b), \quad i=1,2, \ldots, n .
$$

Another set of boundary conditions is obtained from the fact that ruin occurs immediately with zero surplus as

$$
\phi_{L, i}(0 ; b)=1, \quad i=1,2, \ldots, n .
$$

Upon rearrangement of (2.1), division by $h$ and letting $h \rightarrow 0$ yields the integro-differential equations, for $i=1,2, \ldots, n-1$,

$$
\begin{aligned}
c \phi_{L, i}^{\prime}(u ; b)+(\lambda+\gamma+\delta) \phi_{L, i}(u ; b)-\gamma \phi_{L, i+1}(u ; b) & \\
& -\lambda\left(\int_{0}^{b-u} \phi_{L, i}(u+y ; b) p(y) d y+\int_{b-u}^{\infty} \phi_{U, i}(u+y ; b) p(y) d y\right)=0, \quad 0<u<b
\end{aligned}
$$

and

$$
c \phi_{U, i}^{\prime}(u ; b)+(\lambda+\gamma+\delta) \phi_{U, i}(u ; b)-\gamma \phi_{U, i+1}(u ; b)-\lambda \int_{0}^{\infty} \phi_{U, i}(u+y ; b) p(y) d y=0, \quad u<b .
$$


Similarly, (2.2) implies

$$
\begin{aligned}
c \phi_{L, n}^{\prime}(u ; b)+(\lambda+\gamma+\delta) \phi_{L, n}(u ; b)-\gamma \phi_{L, 1}(u ; b) & \\
& -\lambda\left(\int_{0}^{b-u} \phi_{L, n}(u+y ; b) p(y) d y+\int_{b-u}^{\infty} \phi_{U, n}(u+y ; b) p(y) d y\right)=0, \quad 0<u<b,
\end{aligned}
$$

and

$$
c \phi_{U, n}^{\prime}(u ; b)+(\lambda+\gamma+\delta) \phi_{U, n}(u ; b)-\gamma \phi_{U, 1}(b ; b)-\lambda \int_{0}^{\infty} \phi_{U, n}(u+y ; b) p(y) d y=0, \quad u<b .
$$

Although the above derivatives are understood to be left-derivatives, one can apply the same arguments with $u+c h$ in place of $u$ to find that (2.5)-(2.8) are also satisfied by the right-derivatives. Moreover, for $i=1,2, \ldots, n$ the functions $\phi_{L, i}(u ; b)$ and $\phi_{U, i}(u ; b)$ are differentiable within the layers $0<u<b$ and $u>b$ respectively. It is also clear from the above integro-differential equations and the continuity condition (2.3) that smooth pasting condition at $u=b$ indeed holds true, i.e.

$$
\phi_{L, i}^{\prime}(b-; b)=\phi_{U, i}^{\prime}(b+; b), \quad i=1,2, \ldots, n .
$$

It is instructive to note that equations (2.6) and (2.8) only involve $\underline{\phi}_{U}(u ; b)$ but not $\underline{\phi}_{L}(u ; b)$, whereas (2.5) and (2.7) involve both. Therefore, in the next two subsections, we first determine the solution $\underline{\phi}_{U}(u ; b)$ to the partial system which consists of (2.6) and (2.8). In Section 2.4, the solution $\phi_{L}(u ; b)$ to $(2.5)$ and $(2.7)$ is obtained by treating the terms involving $\underline{\phi}_{U}(u ; b)$ as non-homogeneous terms. Any unknown constants involved can finally be determined by the boundary conditions.

\subsection{A representation of $\underline{\phi}_{U}$ via a probabilistic argument}

In this section we adopt a probabilistic approach to determine the solution form of $\phi(u ; b) \equiv \phi_{U, 1}(u ; b)$ for $u \geq b$, which is extended to $\phi_{U, i}(u ; b)$ for $i=1,2, \ldots, n$ as expressed in Lemma 1 below.

Lemma 1. For $i=1,2, \ldots, n$, the solution form of (2.6) and (2.8) is given by

$$
\phi_{U, i}(u ; b)=\left(\frac{\gamma}{\gamma+\delta}\right)^{n-i+1} \phi_{U, 1}(b ; b)+\sum_{j=0}^{n-i} A_{j, n-i+1} u^{j} e^{-\rho_{\gamma+\delta} u}, \quad u \geq b,
$$

where $\rho_{\gamma+\delta}$ is the unique positive root of the equation (in $\xi$ )

$$
c \xi-(\lambda+\gamma+\delta)+\lambda \tilde{p}(\xi)=0,
$$

and $A_{j, k}$ 's are some constants which possibly depend on $b$ but not $u$.

Proof. When the process starts above the level $b$, it will reach level $b$ in the future either because of continuous expenses or because of a dividend payment. Hence, it is important to keep track of the 'Erlang clock' when the surplus reaches $b$ due to one or the other of those two reasons.

We first consider the process $\left\{U_{0}(t)\right\}_{t \geq 0}$ (i.e. where the dividend barrier is set at zero) because the time of ruin of $\left\{U_{0}(t)\right\}_{t \geq 0}$ starting at $U_{0}(0)=u-b \geq 0$ is equivalent to the time to reach level $b$ for the process $\left\{U_{b}(t)\right\}_{t \geq 0}$ starting at $U_{b}(0)=u \geq b$. Note that the process $\left\{U_{0}(t)\right\}_{t \geq 0}$ behaves like $\{U(t)\}_{t \geq 0}$ (the barrier-free model) before time $T_{1}$, and the process $\{U(t)\}_{t \geq 0}$ and the variable $T_{1}$ are independent. In what follows it will be useful to write the $\operatorname{Erlang}(n)$ variable $T_{1}$ as $T_{1}=\sum_{i=1}^{n} X_{i}$, where $\left\{X_{i}\right\}_{i=1}^{n}$ is an i.i.d. sequence of exponential variables each with mean $1 / \gamma$. We define the function, for $k=0,1, \ldots, n-1$,

$$
\sigma_{k+1}(u)=E\left[e^{-\delta \tau} 1\left\{\sum_{i=1}^{k} X_{i} \leq \tau<\sum_{i=1}^{k+1} X_{i}\right\} \mid U(0)=u\right], \quad u \geq 0
$$


as the expected present value of a contingent payment of 1 at the time of ruin $\tau$ for the process $\{U(t)\}_{t \geq 0}$, which is only payable when $\tau$ is between the $\operatorname{Erlang}(k)$ variable $\sum_{i=1}^{k} X_{i}$ and the $\operatorname{Erlang}(k+1)$ variable $\sum_{i=1}^{k+1} X_{i}$. Therefore, returning to the process of interest $\left\{U_{b}(t)\right\}_{t \geq 0}$ with $U_{b}(0)=u \geq b$, the underlying Markovian structure leads to

$$
\phi(u ; b)=\sum_{k=0}^{n-1} \sigma_{k+1}(u-b) \phi_{U, k+1}(b ; b)+E\left[e^{-\delta T_{1}} 1\left\{T_{1}<\tau\right\} \mid U(0)=u-b\right] \phi_{U, 1}(b ; b), \quad u \geq b .
$$

It thus remains to determine (the forms of) $\sigma_{k+1}(u)$ and $E\left[e^{-\delta T_{1}} 1\left\{T_{1}<\tau\right\} \mid U(0)=u\right]$ for $u \geq 0$. Fortunately, these two quantities are available from equations (56) and (40) of Landriault et al. (2012) respectively by noting that the recovery time (denoted by them as $\tau_{0}^{+}$) in the standard risk model is equivalent to the ruin time $\tau$ of the dual model $\{U(t)\}_{t \geq 0}$ apart from a change of sign of the initial level. Quoting their results, we have that

$$
\sigma_{k+1}(u)=\sum_{i=0}^{k} \varpi_{i, k} u^{i} e^{-\rho_{\gamma+\delta} u}, \quad u \geq 0,
$$

and

$$
E\left[e^{-\delta T_{1}} 1\left\{T_{1}<\tau\right\} \mid U(0)=u\right]=\left(\frac{\gamma}{\gamma+\delta}\right)^{n}+\sum_{i=0}^{n-1} \varpi_{i}^{*} u^{i} e^{-\rho_{\gamma+\delta} u}, \quad u \geq 0,
$$

where $\varpi_{i, k}$ 's and $\varpi_{i}^{*}$ 's are constants that do not depend on $u$, and for a given $r>0$ the quantity $\rho_{r}$ is the unique positive root of the Lundberg's equation (in $\xi$ )

$$
c \xi-(\lambda+r)+\lambda \widetilde{p}(\xi)=0 .
$$

Finally, applying (2.13) and (2.14) to (2.12), performing a binomial expansion of $(u-b)^{i}$ and changing the order of summations leads to

$$
\begin{aligned}
\phi(u ; b) & =\sum_{k=0}^{n-1} \sum_{i=0}^{k} \varpi_{i, k}(u-b)^{i} e^{-\rho_{\gamma+\delta}(u-b)} \phi_{U, k+1}(b ; b)+\left[\left(\frac{\gamma}{\gamma+\delta}\right)^{n}+\sum_{i=0}^{n-1} \varpi_{i}^{*}(u-b)^{i} e^{-\rho_{\gamma+\delta}(u-b)}\right] \phi_{U, 1}(b ; b) \\
& =\left(\frac{\gamma}{\gamma+\delta}\right)^{n} \phi_{U, 1}(b ; b)+\sum_{j=0}^{n-1} A_{j} u^{j} e^{-\rho_{\gamma+\delta} u}, \quad u \geq b
\end{aligned}
$$

for some constants $A_{j}$ 's (which possibly depend on $b$ but not $u$ ).

Although the solution form given by $(2.16)$ applies to $\phi(u ; b) \equiv \phi_{U, 1}(u ; b)$ for $u \geq b$, the result can be easily adapted to $\phi_{U, i}(u ; b)$ (for $\left.i=1,2, \ldots, n\right)$. We only need to replace $T_{1}$ by an $\operatorname{Erlang}(n-i+1)$ random variable in the analysis. Then $(2.16)$ still holds true for $\phi_{U, i}(u ; b)$ with $n$ replaced by $n-i+1$. Since $A_{j}$ 's in (2.16) implicitly depend on $n$ as well, the expression (2.10) follows.

Remark 2.1. The root $\rho_{\gamma+\delta}$ corresponds to the notation $\rho_{\gamma}$ in Albrecher et al. (2011a, 2012). However, it is more convenient to adopt the current notation which facilitates the proof of Lemma 2 in Section 3.2.

Remark 2.2. In principle the constants $A_{j}$ 's in (2.16) (and hence $A_{j, n-i+1}$ 's in (2.10)) can be computed in terms of $\varpi_{i, k}$ 's and $\varpi_{i}^{*}$ 's which are available from Landriault et al. (2012). The constants $\varpi_{i, k}$ 's and $\varpi_{i}^{*}$ 's therein are expressed using di Bruno's formula in terms of the Bell polynomial and derivatives of the form $d^{k} \rho_{r} / d r^{k}$. The quantity $d^{k} \rho_{r} / d r^{k}$ can be computed by successive differentiating the Lundberg's equation (2.15) (with $\xi$ replaced by $\rho_{r}$ ) and recursive formula can be obtained.

Remark 2.3. It stems from (2.10) that for $i=1,2, \ldots, n$,

$$
\phi_{U, i}(u ; b) \sim\left(\frac{\gamma}{\gamma+\delta}\right)_{7}^{n-i+1} \phi_{U, 1}(b ; b), \quad u \rightarrow \infty
$$


since $\rho_{\gamma+\delta}>0$. This asymptotic formula intuitively makes sense because starting with a very large initial surplus $U_{b}(0)=u$, it is highly likely that at the next dividend decision time the surplus process $\left\{U_{b}(t)\right\}_{t \geq 0}$ has not ruined yet and is above level $b$. Upon the payment of dividend, the surplus will drop to level $b$ and the Laplace transform of the time remaining until ruin will be $\phi_{U, 1}(b ; b)$ since the 'Erlang clock' restarts. Since the time until the next dividend decision time is distributed as Erlang $(n-i+1)$ which has Laplace transform $[\gamma /(\gamma+\delta)]^{n-i+1}$ (with Laplace transform argument $\delta$ ), this explains the product form of $(2.17)$

\subsection{A recursive solution for $\underline{\phi}_{U}$}

The procedure described in Remark 2.2 can be tedious. We present in Theorem 1 a recursive scheme that allows a direct computation of the constants in Lemma 1 in a more straightforward way.

Theorem 1. For $k=0,1, \ldots, n-1$, the expression

$$
\phi_{U, n-k}(u ; b)=\left(\frac{\gamma}{\gamma+\delta}\right)^{k+1} \phi_{L, 1}(b ; b)+\sum_{j=0}^{k} A_{j, k+1} u^{j} e^{-\rho_{\gamma+\delta} u}, \quad u \geq b,
$$

satisfies (2.3), (2.6) and (2.8), with the coefficients given recursively in $k$ by

$$
A_{j+1, k+1}=\frac{\lambda \sum_{i=j+2}^{k} A_{i, k+1}\left(\begin{array}{l}
i \\
j
\end{array}\right) \int_{0}^{\infty} y^{i-j} e^{-\rho_{\gamma+\delta} y} p(y) d y+\gamma A_{j, k}}{(j+1)\left(c-\lambda \int_{0}^{\infty} y e^{-\rho_{\gamma+\delta} y} p(y) d y\right)}, \quad j=0,1, \ldots, k-1,
$$

and

$$
A_{0, k+1}=e^{\rho_{\gamma+\delta} b}\left[\phi_{L, n-k}(b ; b)-\left(\frac{\gamma}{\gamma+\delta}\right)^{k+1} \phi_{L, 1}(b ; b)-\sum_{j=1}^{k} A_{j, k+1} b^{j} e^{-\rho_{\gamma+\delta} b}\right],
$$

where $\rho_{\gamma+\delta}$ is the unique positive root of (2.11).

Proof. Note that $\phi_{U, n}(u ; b)$ is the only unknown function in $u$ in equation $(2.8)$ since $\phi_{U, 1}(b ; b)$ is just a constant. Thus, one should start with (2.8) to obtain (the coefficients associated to) $\phi_{U, n}(u ; b)$, and then (the coefficients of) $\phi_{U, n-1}(u ; b)$ can be solved from $(2.6)$ by treating $\phi_{U, n}(u ; b)$ as known, and so on. Since we shall work with $\phi_{U, i}(u ; b)$ for decreasing value of $i$, it is more convenient to replace $i$ by $n-k$ in $(2.10)$ so that the above procedure is recursive in $k$. This explains (2.18) where the continuity condition (2.3) at $i=1$ has also been used.

Now it remains to derive the recursive procedures (2.19) and (2.20) by direct substitution. As a starting point (i.e. $k=0$ ), substituting $(2.18)$ at $k=0$ into $(2.8)$ followed by some simple algebra and using the fact that

$$
c \rho_{\gamma+\delta}-(\lambda+\gamma+\delta)+\lambda \widetilde{p}\left(\rho_{\gamma+\delta}\right)=0,
$$

we arrive at $0=0$ which does not yield any information. Utilizing (2.3) at $i=n$ and (2.18) at $k=0$ and $u=b$, equation $(2.20)$ at $k=0$ simply follows by rearrangements. Note that $(2.19)$ does not exist when $k=0$, and hence the case $k=0$ is proved.

Next, for any fixed $k=1,2, \ldots, n-1$, the solution form (2.18) is substituted into (2.6) at $i=n-k$. This yields

$$
\begin{aligned}
& c\left(\sum_{j=0}^{k-1} A_{j+1, k+1}(j+1) u^{j} e^{-\rho_{\gamma+\delta} u}-\rho_{\gamma+\delta} \sum_{j=0}^{k} A_{j, k+1} u^{j} e^{-\rho_{\gamma+\delta} u}\right) \\
&+(\lambda+\gamma+\delta)\left[\left(\frac{\gamma}{\gamma+\delta}\right)^{k+1} \phi_{L, 1}(b ; b)+\sum_{j=0}^{k} A_{j, k+1} u^{j} e^{-\rho_{\gamma+\delta} u}\right]-\gamma\left[\left(\frac{\gamma}{\gamma+\delta}\right)^{k} \phi_{L, 1}(b ; b)+\sum_{j=0}^{k-1} A_{j, k} u^{j} e^{-\rho_{\gamma+\delta} u}\right] \\
&-\lambda\left[\left(\frac{\gamma}{\gamma+\delta}\right)^{k+1} \phi_{L, 1}(b ; b)+\sum_{j=0}^{k}\left(\sum_{i=j}^{k} A_{i, k+1}\left(\begin{array}{c}
i \\
j
\end{array}\right) \int_{0}^{\infty} y^{i-j} e^{-\rho_{\gamma+\delta} y} p(y) d y\right) u^{j} e^{-\rho_{\gamma+\delta} u}=0 .\right.
\end{aligned}
$$


Note that the constant term on the left-hand-side of the above equation equals 0 . The same is also true of the coefficient of $u^{k} e^{-\rho_{\gamma+\delta} u}$ because of (2.21). Equating the coefficients of $u^{j} e^{-\rho_{\gamma+\delta} u}$ for $j=0,1, \ldots, k-1$, rearranging terms and using (2.21) again, one obtains (2.19). Finally, (2.20) can be proved by an application of (2.3) at $i=n-k$ together with $(2.18)$ at $u=b$.

The procedure to compute the coefficients in (2.18) is outlined as follows. First, the baseline case $k=0$ is the simplest case since the coefficient $A_{0,1}$ can be obtained directly from (2.20). Second, for each fixed $k=1,2, \ldots, n-1$, we first put $j=k-1$ into (2.19) which results in an explicit expression for $A_{k, k+1}$ in terms of $A_{k-1, k}$, then we put $j=k-2, k-3, \ldots, 0$ in turn so as to compute $A_{j+1, k+1}$ recursively in terms of $A_{i, k+1}$ 's $(i=j+2, j+3, \ldots, k)$. Finally, the coefficient $A_{0, k+1}$ is computed from (2.20).

\subsection{A solution for $\underline{\phi}_{L}$}

Note that the coefficients of the solution in Theorem 1 regarding the elements of $\phi_{U}(u ; b)$ are expressed in terms of the elements of $\underline{\phi}_{L}(b ; b)$. In what follows we proceed to solve $(2.5)$ and $(2.7)$ for $\underline{\phi}_{L}(u ; b)$. This solution will be in terms of $\underline{\phi}_{U}^{L}(u ; b)$, which in turn depends on $\underline{\phi}_{L}(b ; b)$. These constants will eventually be determined from the boundary conditions (2.4).

To solve equations (2.5) and (2.7), we follow the ideas in Avanzi et al. (2007) to define the variable $z=b-u$ and the functions, for $i=1,2, \ldots, n$,

$$
\varphi_{i}(z)=\phi_{L, i}(b-z ; b)=\phi_{L, i}(u ; b), \quad 0 \leq z \leq b
$$

so that

$$
\phi_{L, i}(b ; b)=\varphi_{i}(0), \quad i=1,2, \ldots, n .
$$

The boundary conditions (2.4) then become

$$
\varphi_{i}(b)=1, \quad i=1,2, \ldots, n .
$$

With the above definition, when $0<z<b$ the equations (2.5) and (2.7) are respectively transformed into

$$
-c \varphi_{i}^{\prime}(z)+(\lambda+\gamma+\delta) \varphi_{i}(z)-\gamma \varphi_{i+1}(z)-\lambda\left(\int_{0}^{z} \varphi_{i}(z-y) p(y) d y+\int_{z}^{\infty} \phi_{U, i}(b-z+y ; b) p(y) d y\right)=0
$$

for $i=1,2, \ldots, n-1$, and

$$
-c \varphi_{n}^{\prime}(z)+(\lambda+\gamma+\delta) \varphi_{n}(z)-\gamma \varphi_{1}(z)-\lambda\left(\int_{0}^{z} \varphi_{n}(z-y) p(y) d y+\int_{z}^{\infty} \phi_{U, n}(b-z+y ; b) p(y) d y\right)=0
$$

As in Avanzi et al. (2007), we extend the domain of the function $\varphi_{i}(z)$ to $z \geq 0$ and define the quantity, for $i=1,2, \ldots, n$,

$$
\alpha_{i}(z)=\int_{z}^{\infty} \phi_{U, i}(b-z+y ; b) p(y) d y, \quad z \geq 0
$$

with Laplace transform

$$
\begin{aligned}
\widetilde{\alpha}_{i}(s) & =\int_{0}^{\infty} e^{-s z} \alpha_{i}(z) d z=\int_{0}^{\infty} e^{-s z} \int_{0}^{\infty} \phi_{U, i}(b+y ; b) p(y+z) d y d z \\
& =\int_{0}^{\infty} \phi_{U, i}(b+y ; b) \int_{0}^{\infty} e^{-s z} p(y+z) d z d y .
\end{aligned}
$$

Taking Laplace transforms on both sides of (2.23) and (2.24) gives

$$
[c s-(\lambda+\gamma+\delta)+\lambda \widetilde{p}(s)] \widetilde{\varphi}_{i}(s)+\gamma \widetilde{\varphi}_{i+1}(s)=c \varphi_{i}(0)-\lambda \widetilde{\alpha}_{i}(s),
$$


for $i=1,2, \ldots, n-1$, and

$$
[c s-(\lambda+\gamma+\delta)+\lambda \widetilde{p}(s)] \widetilde{\varphi}_{n}(s)+\gamma \widetilde{\varphi}_{1}(s)=c \varphi_{n}(0)-\lambda \widetilde{\alpha}_{n}(s) .
$$

In matrix form, (2.26) and (2.27) can be expressed as

$$
\mathbf{A}(s) \underline{\tilde{\varphi}}(s)=c \underline{\varphi}(0)-\lambda \underline{\widetilde{\alpha}}(s),
$$

where

and

$$
\begin{gathered}
\mathbf{A}(s)=[c s-(\lambda+\gamma+\delta)+\lambda \widetilde{p}(s)] \mathbf{I}_{n}+\gamma\left(\begin{array}{ccccc}
0 & 1 & 0 & \cdots & 0 \\
0 & 0 & 1 & \cdots & 0 \\
\vdots & \vdots & \vdots & \ddots & \vdots \\
0 & 0 & 0 & \cdots & 1 \\
1 & 0 & 0 & \cdots & 0
\end{array}\right), \\
\underline{\underline{\varphi}}(s)=\left(\widetilde{\varphi}_{1}(s), \widetilde{\varphi}_{2}(s), \ldots, \widetilde{\varphi}_{n}(s)\right)^{T} \\
\underline{\varphi}(0)=\left(\varphi_{1}(0), \varphi_{2}(0), \ldots, \varphi_{n}(0)\right)^{T}=\underline{\phi}_{L}(b ; b),
\end{gathered}
$$

$$
\underline{\widetilde{\alpha}}(s)=\left(\widetilde{\alpha}_{1}(s), \widetilde{\alpha}_{2}(s), \ldots, \widetilde{\alpha}_{n}(s)\right)^{T} .
$$

Here $\mathbf{I}_{n}$ is the $n$-dimensional identity matrix. When $n=1$, the matrix $\mathbf{A}(s)$ is understood to be the scalar $c s-(\lambda+\delta)+\lambda \widetilde{p}(s)$. From (2.28) it follows that

$$
\underline{\widetilde{\varphi}}(s)=[\mathbf{A}(s)]^{-1}[c \underline{\varphi}(0)-\lambda \underline{\widetilde{\alpha}}(s)] .
$$

Note from $(2.25)$ that the vector $\underline{\widetilde{\alpha}}(s)$ depends on the constant vector $\underline{\phi}_{L}(b ; b)$ in a linear manner through the solution $\phi_{U}(u ; b)$ obtained from Theorem 1, and thus from (2.29) and $(2.30)$ each element of $\tilde{\varphi}(s)$ is linear in $\phi_{L}(b ; \bar{b})$. By analytically (or numerically) inverting (2.30) to obtain $\varphi_{i}(u)$ 's and applying the boundary conditions (2.22), one obtains a system of $n$ linear equations from which the elements of $\underline{\phi}_{L}(b ; b)$ can be solved for. Hence, we arrive at a full characterization of $\left\{\phi_{i}(u ; b) ; u \geq 0\right\}$ for $i=1,2, \ldots, n$.

Remark 2.4. Note that the analytical inversion of (2.30) is possible when each element on the right-hand side of $(2.30)$ can be written as a ratio of two polynomials in $s$. For example, if the jump density $p(\cdot)$ belongs to the class of Erlang mixtures or combinations of exponentials, then it follows from (2.25) that each element of $\widetilde{\varphi}(s)$ can be resolved into partial fractions, which allows analytic inversion.

\section{Expected present value of dividends paid until ruin}

As in Section 2 , we let $V_{i}(u ; b)(i=1,2, \ldots, n)$ be the expected present value of total dividends paid until ruin, given that the initial surplus level is $u$ and the time remaining until the next dividend payment time is distributed as $\operatorname{Erlang}(n-i+1)$.

\subsection{Integro-differential equations}

The same reasoning used to obtain $(2.1)$ and (2.2) leads us to, for $i=1,2, \ldots, n-1$,

$$
V_{i}(u ; b)=[1-(\lambda+\gamma) h] e^{-\delta h} V_{i}(u-c h ; b)+\gamma h e^{-\delta h} V_{i+1}(u-c h ; b)+\lambda h e^{-\delta h} \int_{0}^{\infty} V_{i}(u-c h+y ; b) p(y) d y+o(h),
$$

and

$$
\begin{aligned}
V_{n}(u ; b)= & {[1-(\lambda+\gamma) h] e^{-\delta h} V_{n}(u-c h ; b) } \\
& +\gamma h e^{-\delta h}\left\{V_{1}(u-c h ; b) 1\{u-c h \leq b\}+\left[u-c h-b+V_{1}(b ; b)\right] 1\{u-c h>b\}\right\} \\
& +\lambda h e^{-\delta h} \int_{0}^{\infty} V_{n}(u-c h+y ; b) p(y) d y+o(h),
\end{aligned}
$$


from which the continuity of $V_{i}(u ; b)$ for each $i=1,2, \ldots, n$ can be established by letting $h \rightarrow 0$.

The expected present value of total dividends paid until ruin is then defined piecewise as

$$
V_{i}(u ; b)= \begin{cases}V_{L, i}(u ; b), & 0 \leq u<b . \\ V_{U, i}(u ; b), & u \geq b\end{cases}
$$

Also define $\underline{V}_{L}(u ; b)=\left(V_{L, 1}(u ; b), V_{L, 2}(u ; b), \ldots, V_{L, n}(u ; b)\right)^{T}$ and $\underline{V}_{U}(u ; b)=\left(V_{U, 1}(u ; b), V_{U, 2}(u ; b), \ldots\right.$, $\left.V_{U, n}(u ; b)\right)^{T}$. The continuity at $u=b$ means that

$$
V_{L, i}(b ; b)=V_{U, i}(b ; b), \quad i=1,2, \ldots, n .
$$

Furthermore, if the company has zero surplus, ruin occurs immediately and no dividends will be paid, which yields the boundary conditions

$$
V_{L, i}(0 ; b)=0, \quad i=1,2, \ldots, n .
$$

The integro-differential equations are derived from (3.1) and (3.2) with the same approach as the one used for $(2.5)-(2.8)$. For $i=1,2, \ldots, n-1$, we have

$$
\begin{aligned}
c V_{L, i}^{\prime}(u ; b)+(\lambda+\gamma+\delta) V_{L, i}(u ; b)-\gamma V_{L, i+1}(u ; b) & \\
& -\lambda\left(\int_{0}^{b-u} V_{L, i}(u+y ; b) p(y) d y+\int_{b-u}^{\infty} V_{U, i}(u+y ; b) p(y) d y\right)=0, \quad 0<u<b
\end{aligned}
$$

and

$$
c V_{U, i}^{\prime}(u ; b)+(\lambda+\gamma+\delta) V_{U, i}(u ; b)-\gamma V_{U, i+1}(u ; b)-\lambda \int_{0}^{\infty} V_{U, i}(u+y ; b) p(y) d y=0, \quad u>b .
$$

In addition,

$$
\begin{aligned}
c V_{L, n}^{\prime}(u ; b)+(\lambda & +\gamma+\delta) V_{L, n}(u ; b)-\gamma V_{L, 1}(u ; b) \\
& -\lambda\left(\int_{0}^{b-u} V_{L, n}(u+y ; b) p(y) d y+\int_{b-u}^{\infty} V_{U, n}(u+y ; b) p(y) d y\right)=0, \quad 0<u<b
\end{aligned}
$$

and

$$
c V_{U, n}^{\prime}(u ; b)+(\lambda+\gamma+\delta) V_{U, n}(u ; b)-\gamma\left[u-b+V_{U, 1}(b ; b)\right]-\lambda \int_{0}^{\infty} V_{U, n}(u+y ; b) p(y) d y=0, \quad u>b .
$$

Analogous to (2.9), the continuity condition (3.3) also implies smooth pasting at $u=b$, i.e.

$$
V_{L, i}^{\prime}(b-; b)=V_{U, i}^{\prime}(b+; b), \quad i=1,2, \ldots, n .
$$

Interestingly, similar smooth pasting condition at $u=b$ can also be found in equation (8) of Albrecher et al. (2011a) in which both dividend decisions and the event of ruin are monitored periodically.

For the same reasons as in Section 2, in the next subsection we shall first determine the solution form of the elements of $\underline{V}_{U}(u ; b)$ for the upper layer, and the full solution is characterized in Sections 3.3 and 3.4. Note that the solution form should be different from that of $\underline{\phi}_{U}(u ; b)$, due to the fact that the nonhomogeneous part of (3.8) involves $u$ (in contrast to (2.8) where the non-homogeneous component is a constant).

\subsection{A representation of $\underline{V}_{U}$ via a probabilistic argument}

As in Section 2.2, a probabilistic approach is used in this subsection to determine the solution form of $V(u ; b) \equiv V_{U, 1}(u ; b)$ for $u \geq b$ in the process $\left\{U_{b}(t)\right\}_{t \geq 0}$. We again consider the process $\left\{U_{0}(t)\right\}_{t \geq 0}$ which 
behaves like $\{U(t)\}_{t \geq 0}$ before time $T_{1}$. This leads to

$$
\begin{aligned}
V(u ; b)= & \sum_{k=0}^{n-1} \sigma_{k+1}(u-b) V_{L, k+1}(b ; b)+E\left[e^{-\delta T_{1}} 1\left\{T_{1}<\tau\right\} \mid U(0)=u-b\right] V_{L, 1}(b ; b) \\
& +E\left[e^{-\delta T_{1}} U\left(T_{1}\right) 1\left\{T_{1}<\tau\right\} \mid U(0)=u-b\right], \quad u \geq b .
\end{aligned}
$$

Note that the first two terms represent the potential future dividends at the time when the surplus process $\left\{U_{b}(t)\right\}_{t \geq 0}$ (starting at $u \geq b$ ) hits level $b$ due to either the expense rate or dividend payment. The final term in the above expression arises because a dividend payment of $U_{b}\left(T_{1}\right)-b$ is made at time $T_{1}$ if $\left\{U_{b}(t)\right\}_{t \geq 0}$ has not downcrossed level $b$ in the interim. The expression (3.10) can be rewritten in a more tractable form, which is the object of the following lemma.

Lemma 2. For $i=1,2, \ldots, n$, the solution form of (3.6) and (3.8) is given by

$$
\begin{aligned}
V_{U, i}(u ; b)= & \left(\frac{\gamma}{\gamma+\delta}\right)^{n-i+1}\left(\frac{(n-i+1)(\lambda \mu-c)}{\gamma+\delta}+V_{L, 1}(b ; b)-b\right)+\left(\frac{\gamma}{\gamma+\delta}\right)^{n-i+1} u \\
& +\sum_{j=0}^{n-i} B_{j, n-i+1} u^{j} e^{-\rho_{\gamma+\delta} u}, \quad u \geq b,
\end{aligned}
$$

where $\rho_{\gamma+\delta}$ is the unique positive root of (2.11) and $B_{j, k}$ 's are some constants which possibly depend on $b$ but not $u$.

Proof. Our starting point is (3.10). Since the forms of $\sigma_{k+1}(u)$ and $E\left[e^{-\delta T_{1}} 1\left\{T_{1}<\tau\right\} \mid U(0)=u\right]$ for $u \geq 0$ are already given by (2.13) and (2.14), we first focus on the determination of $E\left[e^{-\delta T_{1}} U\left(T_{1}\right) 1\left\{T_{1}<\right.\right.$ $\tau\} \mid U(0)=u]$ for $u \geq 0$.

We shall need some auxiliary results from the literature. In the dual model $\{U(t)\}_{t \geq 0}$, the Laplace transform of the time to ruin is given by (with a slight abuse of notation)

$$
\phi(u ; \infty)=E\left[e^{-\delta \tau} 1\{\tau<\infty\} \mid U(0)=u\right]=e^{-\rho_{\delta} u}, \quad u \geq 0,
$$

where $\rho_{\delta}$ is defined in the same way as in (2.15); see e.g. $\mathrm{Ng}$ (2009, Lemma 1). Note that given an initial surplus of $u=U(0) \geq 0$, the distribution of $\tau$ consists of a point mass at $u / c$ with probability $e^{-\lambda(u / c)}$ since if no gain occurs within the period $[0, u / c]$ then ruin occurs at time $u / c$ due to the expense rate. The density part of $\tau$ at $t$ will be denoted by $h_{u}(t)$ for $t \geq 0$. Note also that $h_{u}(t)=0$ for $0 \leq t \leq u / c$. Hence, the Laplace transform (3.12) can be represented as

$$
\phi(u ; \infty)=\int_{0}^{\infty} e^{-\delta t} h_{u}(t) d t+e^{-\delta\left(\frac{u}{c}\right)} e^{-\lambda\left(\frac{u}{c}\right)}=\widetilde{h}_{u}(\delta)+e^{-(\lambda+\delta)\left(\frac{u}{c}\right)}, \quad u \geq 0 .
$$

To study $E\left[e^{-\delta T_{1}} U\left(T_{1}\right) 1\left\{T_{1}<\tau\right\} \mid U(0)=u\right]$, we shall look at $\sum_{i=1}^{N\left(T_{1}\right)} Y_{i}-c T_{1}$ which is the increment of the process $\{U(t)\}_{t \geq 0}$ from time 0 to time $T_{1}$. Due to the discounting from time $T_{1}$ to 0 , we consider the joint Laplace transform of $\left(T_{1}, \sum_{i=1}^{N\left(T_{1}\right)} Y_{i}-c T_{1}\right)$ which can be represented as

$$
E\left[e^{-\delta T_{1}-s\left(\sum_{i=1}^{N\left(T_{1}\right)} Y_{i}-c T_{1}\right)}\right]=\left(\frac{\gamma}{\gamma+\delta-c s+\lambda[1-\widetilde{p}(s)]}\right)^{n}=\int_{-\infty}^{\infty} e^{-s y} g_{\delta}(y) d y,
$$

where

$$
g_{\delta}(y)=g_{\delta,-}(-y) 1\{y<0\}+g_{\delta,+}(y) 1\{y>0\}, \quad-\infty<y<\infty
$$

see Albrecher et al. (2011a, 2012). Here $g_{\delta}(\cdot)$ is the discounted density of the increment of the process $\{U(t)\}_{t \geq 0}$ observed at time $T_{1}$, with $g_{\delta,+}(\cdot)$ and $g_{\delta,-}(\cdot)$ representing the cases of net gain and net loss 
respectively. In particular, the (defective) density of $\sum_{i=1}^{N(t)} Y_{i}-c t$ at $y$ when $\sum_{i=1}^{N(t)} Y_{i}-c t>0$, namely $g_{+}(y, t)$, satisfies

$$
g_{\delta,+}(y)=\int_{0}^{\infty} e^{-\delta t} g_{+}(y, t) \frac{\gamma^{n} t^{n-1} e^{-\gamma t}}{(n-1) !} d t=\int_{0}^{\infty} \frac{\gamma^{n} e^{-(\gamma+\delta) t} t^{n-1}}{(n-1) !} g_{+}(y, t) d t, \quad y>0 .
$$

Another auxiliary result from Albrecher et al. (2011a) is that $g_{\delta,-}(\cdot)$ is indeed a (defective) mixture of Erlangs with the same scale parameter $\rho_{\gamma+\delta}$ such that

$$
g_{\delta,-}(y)=\sum_{k=1}^{n} q_{k} \frac{\rho_{\gamma+\delta}^{k} y^{k-1} e^{-\rho_{\gamma+\delta} y}}{(k-1) !}, \quad y>0
$$

for some constants $q_{k}$ 's.

Now, we can evaluate $E\left[e^{-\delta T_{1}} U\left(T_{1}\right) 1\left\{T_{1}<\tau\right\} \mid U(0)=u\right]$ as

$$
\begin{aligned}
& E\left[e^{-\delta T_{1}} U\left(T_{1}\right) 1\left\{T_{1}<\tau\right\} \mid U(0)=u\right] \\
= & \int_{0}^{u} y g_{\delta,-}(u-y) d y+\int_{u}^{\infty} y g_{\delta,+}(y-u) d y \\
& -\int_{0}^{\infty} \int_{0}^{\infty} e^{-\delta t} y\left(\int_{0}^{t} h_{u}(x) g_{+}(y, t-x) d x+1\left\{t>\frac{u}{c}\right\} e^{-\lambda\left(\frac{u}{c}\right)} g_{+}\left(y, t-\frac{u}{c}\right)\right) \frac{\gamma^{n} t^{n-1} e^{-\gamma t}}{(n-1) !} d y d t .
\end{aligned}
$$

The above equation is decomposed as follows. The first integral represents the case where at time $T_{1}$ a net loss of $u-y$ (where $0<y<u$ ) is suffered by the company, and hence $U\left(T_{1}\right)=y$. Similarly, the second integral is the case where a net gain of $y-u$ (where $y>u$ ) is earned by the company and therefore $U\left(T_{1}\right)=y$. However, the above integrals include the sample paths in which ruin of $\{U(t)\}_{t \geq 0}$ has occurred before time $T_{1}$ (i.e. $\tau<T_{1}$ ) but the process is at a positive level at time $T_{1}$, and these contributions have to be removed. This can be explained by the third integral, which is obtained by first conditioning on $T_{1}=t$. Given $T_{1}=t$, if ruin occurs at some time $x(0<x<t)$ at which $\{U(t)\}_{t \geq 0}$ reaches level 0 for the first time, and in $t-x$ units of time starting from time $x$ the process $\{U(t)\}_{t \geq 0}$ increases by level $y$, then the contribution of $e^{-\delta t} y$ should be removed. Noting that the distribution of $\tau$ consists of both a density part as well as a point mass, the above argument leads to the third integral in (3.17).

We now aim at simplifying (3.17). By substitution of (3.16), the first integral in (3.17) becomes

$$
\begin{aligned}
\int_{0}^{u} y g_{\delta,-}(u-y) d y & =\int_{0}^{u}(u-y) \sum_{k=1}^{n} q_{k} \frac{\rho_{\gamma+\delta}^{k} y^{k-1} e^{-\rho_{\gamma+\delta} y}}{(k-1) !} d y \\
& =\sum_{k=1}^{n} q_{k}\left(u \int_{0}^{u} \frac{\rho_{\gamma+\delta}^{k} y^{k-1} e^{-\rho_{\gamma+\delta} y}}{(k-1) !} d y-\frac{k}{\rho_{\gamma+\delta}} \int_{0}^{u} \frac{\rho_{\gamma+\delta}^{k+1} y^{k} e^{-\rho_{\gamma+\delta} y}}{k !} d y\right) \\
& =\sum_{k=1}^{n} q_{k}\left[u\left(1-\sum_{i=0}^{k-1} e^{-\rho_{\gamma+\delta} u} \frac{\left(\rho_{\gamma+\delta} u\right)^{i}}{i !}\right)-\frac{k}{\rho_{\gamma+\delta}}\left(1-\sum_{i=0}^{k} e^{-\rho_{\gamma+\delta} u} \frac{\left(\rho_{\gamma+\delta} u\right)^{i}}{i !}\right)\right] \\
& =u \sum_{k=1}^{n} q_{k}-\sum_{k=1}^{n} \frac{k q_{k}}{\rho_{\gamma+\delta}}+\sum_{k=1}^{n} q_{k} \sum_{i=0}^{k-1} \frac{(k-i) \rho_{\gamma+\delta}^{i-1}}{i !} u^{i} e^{-\rho_{\gamma+\delta} u} \\
& =u \int_{0}^{\infty} g_{\delta,-}(y) d y-\int_{0}^{\infty} y g_{\delta,-}(y) d y+\sum_{j=0}^{n-1} \zeta_{j} u^{j} e^{-\rho_{\gamma+\delta} u},
\end{aligned}
$$

for some constants $\zeta_{j}$ 's that do not depend on $u$, and where the last equality again follows from the mixed Erlang representation (3.16) and a change of order of summations. The second integral in (3.17) is simply given by

$$
\int_{u}^{\infty} y g_{\delta,+}(y-u) d y=u \int_{0}^{\infty} g_{\delta,+}(y) d y+\int_{0}^{\infty} y g_{\delta,+}(y) d y .
$$


The third integral of (3.17) can be rewritten as

$$
\begin{aligned}
& \int_{0}^{\infty} \int_{0}^{\infty} e^{-\delta t} y\left(\int_{0}^{t} h_{u}(x) g_{+}(y, t-x) d x+1\left\{t>\frac{u}{c}\right\} e^{-\lambda\left(\frac{u}{c}\right)} g_{+}\left(y, t-\frac{u}{c}\right)\right) \frac{\gamma^{n} t^{n-1} e^{-\gamma t}}{(n-1) !} d y d t \\
= & \gamma^{n} \int_{0}^{\infty} y\left[\int_{0}^{\infty} \frac{e^{-(\gamma+\delta) t} t^{n-1}}{(n-1) !}\left(\int_{0}^{t} h_{u}(x) g_{+}(y, t-x) d x\right) d t+e^{-\lambda\left(\frac{u}{c}\right)} \int_{\frac{u}{c}}^{\infty} \frac{e^{-(\gamma+\delta) t} t^{n-1}}{(n-1) !} g_{+}\left(y, t-\frac{u}{c}\right) d t\right] d y .
\end{aligned}
$$

The first integral inside the square bracket of the above expression admits the representation

$$
\begin{aligned}
& \int_{0}^{\infty} \frac{e^{-(\gamma+\delta) t} t^{n-1}}{(n-1) !}\left(\int_{0}^{t} h_{u}(x) g_{+}(y, t-x) d x\right) d t \\
= & \left.\frac{(-1)^{n-1}}{(n-1) !} \frac{d^{n-1}}{d r^{n-1}}\left[\int_{0}^{\infty} e^{-r t}\left(\int_{0}^{t} h_{u}(x) g_{+}(y, t-x) d x\right) d t\right]\right|_{r=\gamma+\delta} \\
= & \left.\frac{(-1)^{n-1}}{(n-1) !} \frac{d^{n-1}}{d r^{n-1}}\left[\widetilde{h}_{u}(r) \widetilde{g}_{+}(y, r)\right]\right|_{r=\gamma+\delta} \\
= & (-1)^{n-1} \sum_{k=0}^{n-1}\left(\left.\frac{1}{k !} \frac{d^{k}}{d r^{k}} \widetilde{h}_{u}(r)\right|_{r=\gamma+\delta}\right)\left(\left.\frac{1}{(n-1-k) !} \frac{d^{n-1-k}}{d r^{n-1-k}} \widetilde{g}_{+}(y, r)\right|_{r=\gamma+\delta}\right),
\end{aligned}
$$

where $\widetilde{g}_{+}(y, r)=\int_{0}^{\infty} e^{-r t} g_{+}(y, t) d t$ and the Leibniz Rule for differentiating product has been used. Next, the second integral inside the square bracket of (3.20) is

$$
\begin{aligned}
& e^{-\lambda\left(\frac{u}{c}\right)} \int_{\frac{u}{c}}^{\infty} \frac{e^{-(\gamma+\delta) t} t^{n-1}}{(n-1) !} g_{+}\left(y, t-\frac{u}{c}\right) d t=e^{-\lambda\left(\frac{u}{c}\right)} \int_{0}^{\infty} \frac{e^{-(\gamma+\delta)\left(t+\frac{u}{c}\right)}\left(t+\frac{u}{c}\right)^{n-1}}{(n-1) !} g_{+}(y, t) d t \\
= & \sum_{k=0}^{n-1} e^{-(\lambda+\gamma+\delta)\left(\frac{u}{c}\right)} \frac{\left(\frac{u}{c}\right)^{k}}{k !} \int_{0}^{\infty} \frac{e^{-(\gamma+\delta) t} t^{n-1-k}}{(n-1-k) !} g_{+}(y, t) d t \\
= & (-1)^{n-1} \sum_{k=0}^{n-1}\left(\left.\frac{1}{k !} \frac{d^{k}}{d r^{k}} e^{-(\lambda+r)\left(\frac{u}{c}\right)}\right|_{r=\gamma+\delta}\right)\left(\left.\frac{1}{(n-1-k) !} \frac{d^{n-1-k}}{d r^{n-1-k}} \widetilde{g}_{+}(y, r)\right|_{r=\gamma+\delta}\right) .
\end{aligned}
$$

Adding (3.21) and (3.22) followed by application of (3.12) and (3.13), (3.20) is reduced to

$$
\begin{aligned}
& \int_{0}^{\infty} \int_{0}^{\infty} e^{-\delta t} y\left(\int_{0}^{t} h_{u}(x) g_{+}(y, t-x) d x+1\left\{t>\frac{u}{c}\right\} e^{-\lambda\left(\frac{u}{c}\right)} g_{+}\left(y, t-\frac{u}{c}\right)\right) \frac{\gamma^{n} t^{n-1} e^{-\gamma t}}{(n-1) !} d y d t \\
= & \gamma^{n}(-1)^{n-1} \sum_{k=0}^{n-1}\left(\left.\frac{1}{k !} \frac{d^{k}}{d r^{k}} e^{-\rho_{r} u}\right|_{r=\gamma+\delta}\right) \int_{0}^{\infty} y\left(\left.\frac{1}{(n-1-k) !} \frac{d^{n-1-k}}{d r^{n-1-k}} \widetilde{g}_{+}(y, r)\right|_{r=\gamma+\delta}\right) d y .
\end{aligned}
$$

Inductively (or by di Bruno's formula in connection to Remark 2.2), it can be seen that

$$
\left.\frac{d^{k}}{d r^{k}} e^{-\rho_{r} u}\right|_{r=\gamma+\delta}=\sum_{i=0}^{k} \chi_{i, k} u^{i} e^{-\rho_{\gamma+\delta} u}, \quad k=0,1, \ldots,
$$

for some coefficients $\chi_{i, k}$ 's that do not depend on $u$. By further noting that the integral on the right-hand side of (3.23) does not depend on $u$, one asserts that (3.23) admits the representation

$$
\begin{aligned}
& \int_{0}^{\infty} \int_{0}^{\infty} e^{-\delta t} y\left(\int_{0}^{t} h_{u}(x) g_{+}(y, t-x) d x+1\left\{t>\frac{u}{c}\right\} e^{-\lambda\left(\frac{u}{c}\right)} g_{+}\left(y, t-\frac{u}{c}\right)\right) \frac{\gamma^{n} t^{n-1} e^{-\gamma t}}{(n-1) !} d y d t \\
= & \sum_{j=0}^{n-1} \zeta_{j}^{*} u^{j} e^{-\rho_{\gamma+\delta} u}
\end{aligned}
$$


for some constants $\zeta_{j}^{*}$ 's.

We are then able to simplify (3.17) by combining (3.18), (3.19) and (3.24). First, with (3.14) at $s=0$ and (3.15), one has that

$$
u \int_{0}^{\infty} g_{\delta,-}(y) d y+u \int_{0}^{\infty} g_{\delta,+}(y) d y=u \int_{-\infty}^{\infty} g_{\delta}(y) d y=u E\left[e^{-\delta T_{1}}\right]=\left(\frac{\gamma}{\gamma+\delta}\right)^{n} u .
$$

Second,

$$
\begin{aligned}
\int_{0}^{\infty} y g_{\delta,+}(y) d y-\int_{0}^{\infty} y g_{\delta,-}(y) d y & =\int_{-\infty}^{\infty} y g_{\delta}(y) d y=-\left.\frac{d}{d s}\left(\frac{\gamma}{\gamma+\delta-c s+\lambda[1-\widetilde{p}(s)]}\right)^{n}\right|_{s=0} \\
& =\left(\frac{\gamma}{\gamma+\delta}\right)^{n} \frac{n(\lambda \mu-c)}{\gamma+\delta}
\end{aligned}
$$

Using the above two expressions, (3.17) can be written in the form

$$
E\left[e^{-\delta T_{1}} U\left(T_{1}\right) 1\left\{T_{1}<\tau\right\} \mid U(0)=u\right]=\left(\frac{\gamma}{\gamma+\delta}\right)^{n} \frac{n(\lambda \mu-c)}{\gamma+\delta}+\left(\frac{\gamma}{\gamma+\delta}\right)^{n} u+\sum_{j=0}^{n-1} \eta_{j} u^{j} e^{-\rho_{\gamma+\delta} u}
$$

for some constants $\eta_{j}$ 's.

Finally, application of (2.13), (2.14) and (3.25) to (3.10) yields

$$
\begin{aligned}
V(u ; b)= & \sum_{k=0}^{n-1} \sum_{i=0}^{k} \varpi_{i, k}(u-b)^{i} e^{-\rho_{\gamma+\delta}(u-b)} V_{L, k+1}(b ; b)+\left[\left(\frac{\gamma}{\gamma+\delta}\right)^{n}+\sum_{i=0}^{n-1} \varpi_{i}^{*}(u-b)^{i} e^{-\rho_{\gamma+\delta}(u-b)}\right] V_{L, 1}(b ; b) \\
& +\left(\frac{\gamma}{\gamma+\delta}\right)^{n} \frac{n(\lambda \mu-c)}{\gamma+\delta}+\left(\frac{\gamma}{\gamma+\delta}\right)^{n}(u-b)+\sum_{j=0}^{n-1} \eta_{j}(u-b)^{j} e^{-\rho_{\gamma+\delta}(u-b)} \\
= & \left(\frac{\gamma}{\gamma+\delta}\right)^{n}\left(\frac{n(\lambda \mu-c)}{\gamma+\delta}+V_{L, 1}(b ; b)-b\right)+\left(\frac{\gamma}{\gamma+\delta}\right)^{n} u+\sum_{j=0}^{n-1} B_{j} u^{j} e^{-\rho_{\gamma+\delta} u}, \quad u \geq b,
\end{aligned}
$$

for some constants $B_{j}$ 's (which possibly depend on $b$ but not $u$ ). Analogous to (2.10), the above result concerning $V(u ; b) \equiv V_{U, 1}(u ; b)$ for $u \geq b$ can be extended to (3.11) as stated in Lemma 2 .

Remark 3.1. In principle, one may consider computing the constants $B_{j}$ 's (and hence $B_{j, n-i+1}$ 's) directly. However, this will not only involve derivatives of the form $d^{k} \rho_{r} / d r^{k}$ (see Remark 2.2) but also $d^{k} \widetilde{g}_{+}(y, r) / d r^{k}$ (see (3.23)). Explicit expression for the latter quantity is not easy to obtain in general, and therefore this further justifies our approach of back substituting (3.11) into the integro-differential equations (3.6) and (3.8) so that we can directly work with the coefficients $B_{j, n-i+1}$ 's.

Remark 3.2. It follows from Lemma 2 that

$$
V_{U, i}(u ; b) \sim\left(\frac{\gamma}{\gamma+\delta}\right)^{n-i+1} u, \quad u \rightarrow \infty,
$$

for $i=1,2, \ldots, n$. This asymptotic formula can again be explained in a similar way to (2.17): even if the surplus level $U_{b}(0)=u$ is very large, it is highly likely that a dividend will be paid at the next dividend decision time and the surplus will drop to level $b$. Any additional initial surplus $u$ will not affect the surplus process itself, but instead it will increase the next dividend payment by the same amount. The value of potential future dividends is negligible compared to this first payment. The term $[\gamma /(\gamma+\delta)]^{n-i+1}$ represents the expected present value (at discount rate $\delta$ ) of a dollar paid at the next dividend payment time because the remaining time until the next dividend decision time is distributed as $\operatorname{Erlang}(n-i+1)$. 


\subsection{A recursive solution for $\underline{V}_{U}$}

By an application of the solution form (3.11) of each element of $\underline{V}_{U}(u ; b)$, the following Theorem can be proved in an identical manner as Theorem 1 and the proof is omitted.

Theorem 2. For $k=0,1, \ldots, n-1$, the expression

$$
\begin{aligned}
V_{U, n-k}(u ; b)= & \left(\frac{\gamma}{\gamma+\delta}\right)^{k+1}\left(\frac{(k+1)(\lambda \mu-c)}{\gamma+\delta}+V_{L, 1}(b ; b)-b\right)+\left(\frac{\gamma}{\gamma+\delta}\right)^{k+1} u \\
& +\sum_{j=0}^{k} B_{j, k+1} u^{j} e^{-\rho_{\gamma+\delta} u}, \quad u \geq b,
\end{aligned}
$$

satisfies (3.3), (3.6) and (3.8), with the coefficients given recursively in $k$ by

$$
B_{j+1, k+1}=\frac{\lambda \sum_{i=j+2}^{k} B_{i, k+1}\left(\begin{array}{l}
i \\
j
\end{array}\right) \int_{0}^{\infty} y^{i-j} e^{-\rho_{\gamma+\delta} y} p(y) d y+\gamma B_{j, k}}{(j+1)\left(c-\lambda \int_{0}^{\infty} y e^{-\rho_{\gamma+\delta} y} p(y) d y\right)}, \quad j=0,1, \ldots, k-1,
$$

and

$$
\begin{aligned}
B_{0, k+1}=e^{\rho_{\gamma+\delta} b} & {\left[V_{L, n-k}(b ; b)-\left(\frac{\gamma}{\gamma+\delta}\right)^{k+1}\left(\frac{(k+1)(\lambda \mu-c)}{\gamma+\delta}+V_{L, 1}(b ; b)-b\right)\right.} \\
& \left.-\left(\frac{\gamma}{\gamma+\delta}\right)^{k+1} b-\sum_{j=1}^{k} B_{j, k+1} b^{j} e^{-\rho_{\gamma+\delta} b}\right],
\end{aligned}
$$

where $\rho_{\gamma+\delta}$ is the unique positive root of (2.11).

\subsection{A solution for $\underline{V}_{L}$}

In this section, we solve (3.5) and (3.7) for $\underline{V}_{L}(u ; b)$ which will lead to a full solution to $\left\{V_{i}(u ; b) ; u \geq 0\right\}$ for $i=1,2, \ldots, n$. Since the procedures of solving (3.5) and (3.7) are identical to those used for solving (2.5) and (2.7), we simply state the main results here for sake of brevity. By defining the variable $z=b-u$ and the function, for $i=1,2, \ldots, n$,

$$
\nu_{i}(z)=V_{L, i}(b-z ; b)=V_{L, i}(u ; b), \quad 0 \leq z \leq b,
$$

and the boundary conditions (3.4) become

$$
\nu_{i}(b)=0, \quad i=1,2, \ldots, n .
$$

Again we extend the domain of the function $\nu_{i}(z)$ to $z \geq 0$. By defining the auxiliary function, for $i=$ $1,2, \ldots, n$,

$$
\vartheta_{i}(z)=\int_{z}^{\infty} V_{U, i}(b-z+y ; b) p(y) d y, \quad z \geq 0,
$$

together with the column vectors

$$
\begin{gathered}
\underline{\widetilde{\nu}}(s)=\left(\widetilde{\nu}_{1}(s), \widetilde{\nu}_{2}(s), \ldots, \widetilde{\nu}_{n}(s)\right)^{T}, \\
\underline{\nu}(0)=\left(\nu_{1}(0), \nu_{2}(0), \ldots, \nu_{n}(0)\right)^{T}=\underline{V}_{L}(b ; b),
\end{gathered}
$$

and

we arrive at

$$
\underline{\widetilde{\vartheta}}(s)=\left(\widetilde{\vartheta}_{1}(s), \widetilde{\vartheta}_{2}(s), \ldots, \widetilde{\vartheta}_{n}(s)\right)^{T},
$$

$$
\underline{\widetilde{v}}(s)=[\mathbf{A}(s)]^{-1}[c \underline{\nu}(0)-\lambda \underline{\widetilde{\vartheta}}(s)] .
$$

It is clear that the vector $\underline{\widetilde{\vartheta}}(s)$ is linear in $\underline{V}_{L}(b ; b)$ through the solution $\underline{V}_{U}(u ; b)$ obtained in Theorem 2 , and hence from (3.27) and (3.28) each element of $\underline{\underline{v}}(s)$ is linear in $\underline{V}_{L}(b ; b)$. Inverting the Laplace transforms in (3.28) together with the application of the boundary conditions (3.26) leads to a system of $n$ linear equations in $\underline{V}_{L}(b ; b)$. Finally, $\left\{V_{i}(u ; b) ; u \geq 0\right\}$ for $i=1,2, \ldots, n$ is fully characterized. 


\section{Explicit solutions when gains and inter-dividend-decision times are both exponential}

In previous sections, the gain distribution has been kept general (except that the gain should possess rational Laplace transform in order to perform various Laplace transform inversions analytically), and the intervals between successive dividend decision times were generally Erlang $(n)$ distributed. However, when the inter-dividend-decision times are exponential with mean $1 / \gamma($ i.e. $n=1)$ and the gains are exponential with mean $1 / \beta$ (where $\beta>0$ ), simplifications occur and nice explicit solutions for $\phi(u ; b) \equiv \phi_{1}(u ; b)$ and $V(u ; b) \equiv V_{1}(u ; b)$ are available.

\subsection{Solution for $\phi$}

First, $\phi(u ; b)$ can be derived using the algorithm in Sections 2.3 and 2.4. But alternatively, in such a simple situation one can also solve the integro-differential equations (2.7) and (2.8) directly subject to the boundary conditions (2.3) and (2.4). (Note that (2.5) and (2.6) are non-existent when $n=1$.) This typically involves an application of the operator $(d / d u-\beta)$ to reduce $(2.7)$ and (2.8) to ordinary differential equations with constant coefficients. Omitting the tedious but straightforward algebra, with either method we ultimately arrive at

$$
\begin{aligned}
& \phi_{L}(u ; b)=\phi_{L, 1}(u ; b) \\
&= \frac{\left[\beta \delta\left(\rho_{\gamma+\delta}+\alpha_{2}\right)+\gamma \alpha_{2}\left(\beta+\rho_{\gamma+\delta}\right)\right]\left(\beta-\alpha_{1}\right) e^{\alpha_{2} b+\alpha_{1} u}-\left[\beta \delta\left(\rho_{\gamma+\delta}+\alpha_{1}\right)+\gamma \alpha_{1}\left(\beta+\rho_{\gamma+\delta}\right)\right]\left(\beta-\alpha_{2}\right) e^{\alpha_{1} b+\alpha_{2} u}}{\left[\beta \delta\left(\rho_{\gamma+\delta}+\alpha_{2}\right)+\gamma \alpha_{2}\left(\beta+\rho_{\gamma+\delta}\right)\right]\left(\beta-\alpha_{1}\right) e^{\alpha_{2} b}-\left[\beta \delta\left(\rho_{\gamma+\delta}+\alpha_{1}\right)+\gamma \alpha_{1}\left(\beta+\rho_{\gamma+\delta}\right)\right]\left(\beta-\alpha_{2}\right) e^{\alpha_{1} b}}, \\
& 0 \leq u \leq b,
\end{aligned}
$$

and

$$
\begin{aligned}
& \phi_{U}(u ; b)=\phi_{U, 1}(u ; b) \\
&= \frac{\beta \delta\left(\beta+\rho_{\gamma+\delta}\right)\left(\alpha_{1}-\alpha_{2}\right) e^{\left(\alpha_{1}+\alpha_{2}\right) b} e^{-\rho_{\gamma+\delta}(u-b)}}{\left[\beta \delta\left(\rho_{\gamma+\delta}+\alpha_{1}\right)+\gamma \alpha_{1}\left(\beta+\rho_{\gamma+\delta}\right)\right]\left(\beta-\alpha_{2}\right) e^{\alpha_{1} b}-\left[\beta \delta\left(\rho_{\gamma+\delta}+\alpha_{2}\right)+\gamma \alpha_{2}\left(\beta+\rho_{\gamma+\delta}\right)\right]\left(\beta-\alpha_{1}\right) e^{\alpha_{2} b}}+\frac{\gamma}{\gamma+\delta} \phi_{L}(b ; b), \\
& u \geq b,
\end{aligned}
$$

where $\alpha_{1}$ and $\alpha_{2}$ are the roots of the quadratic equation (in $\xi$ )

$$
c \xi^{2}+(\lambda+\delta-\beta c) \xi-\delta \beta=0 .
$$

It is instructive to note that the above equation is equivalent to equation (3.4) in Avanzi et al. (2007). This means that the roots $\alpha_{1}$ and $\alpha_{2}$ are the same as those in the classical dual model.

Remark 4.1. Because $\rho_{\gamma+\delta} \rightarrow \infty$ as $\gamma \rightarrow \infty$, at the limit one has

$$
\begin{aligned}
& \lim _{\gamma \rightarrow \infty} \phi_{L}(u ; b)=\frac{\alpha_{2}\left(\beta-\alpha_{1}\right) e^{\alpha_{2} b+\alpha_{1} u}-\alpha_{1}\left(\beta-\alpha_{2}\right) e^{\alpha_{1} b+\alpha_{2} u}}{\alpha_{2}\left(\beta-\alpha_{1}\right) e^{\alpha_{2} b}-\alpha_{1}\left(\beta-\alpha_{2}\right) e^{\alpha_{1} b}}=\frac{\left(\beta-\alpha_{1}-\frac{\lambda}{c}\right) e^{-\alpha_{1}(b-u)}-\left(\beta-\alpha_{2}-\frac{\lambda}{c}\right) e^{-\alpha_{2}(b-u)}}{\left(\beta-\alpha_{1}-\frac{\lambda}{c}\right) e^{-\alpha_{1} b}-\left(\beta-\alpha_{2}-\frac{\lambda}{c}\right) e^{-\alpha_{2} b}}, \\
& 0 \leq u \leq b,
\end{aligned}
$$

where the last equality follows because $\alpha_{i}\left(\beta-\alpha_{i}-\lambda / c\right) /\left(\beta-\alpha_{i}\right)=-\delta / c$ as $\alpha_{i}$ satisfies (4.1) for $i=1,2$. The last expression is consistent with that in Cheung and Drekic (2008, Section 4.2) (apart from some notational changes). This is expected since the limit $\gamma \rightarrow \infty$ corresponds to the dual model under the classical barrier strategy. 


\subsection{Solution for $V$}

Similarly, for the expected discounted dividends until ruin $V(u ; b)$ one has

$$
\begin{aligned}
& V_{L}(u ; b)=V_{L, 1}(u ; b) \\
&= \frac{c \rho_{\gamma+\delta}^{2} \gamma\left(\beta-\alpha_{1}\right)\left(\beta-\alpha_{2}\right)\left(e^{\alpha_{1} u}-e^{\alpha_{2} u}\right)}{(\gamma+\delta) \beta\left\{\left[\beta \delta\left(\rho_{\gamma+\delta}+\alpha_{1}\right)+\gamma \alpha_{1}\left(\beta+\rho_{\gamma+\delta}\right)\right]\left(\beta-\alpha_{2}\right) e^{\alpha_{1} b}-\left[\beta \delta\left(\rho_{\gamma+\delta}+\alpha_{2}\right)+\gamma \alpha_{2}\left(\beta+\rho_{\gamma+\delta}\right)\right]\left(\beta-\alpha_{1}\right) e^{\alpha_{2} b}\right\}}, \\
& 0 \leq u \leq b, \quad(4.2)
\end{aligned}
$$

and

$$
\begin{aligned}
& V_{U}(u ; b)=V_{U, 1}(u ; b) \\
= & \frac{c \gamma\left(\beta+\rho_{\gamma+\delta}\right)\left[\alpha_{1}^{2}\left(\beta-\alpha_{2}\right) e^{\alpha_{1} b}-\alpha_{2}^{2}\left(\beta-\alpha_{1}\right) e^{\alpha_{2} b}\right] e^{-\rho_{\gamma+\delta}(u-b)}}{(\gamma+\delta) \beta\left\{\left[\beta \delta\left(\rho_{\gamma+\delta}+\alpha_{1}\right)+\gamma \alpha_{1}\left(\beta+\rho_{\gamma+\delta}\right)\right]\left(\beta-\alpha_{2}\right) e^{\alpha_{1} b}-\left[\beta \delta\left(\rho_{\gamma+\delta}+\alpha_{2}\right)+\gamma \alpha_{2}\left(\beta+\rho_{\gamma+\delta}\right)\right]\left(\beta-\alpha_{1}\right) e^{\alpha_{2} b}\right\}} \\
& +\frac{\gamma}{\gamma+\delta}\left(\frac{\frac{\lambda}{\beta}-c}{\gamma+\delta}+V_{L}(b ; b)+u-b\right), \quad u \geq b .
\end{aligned}
$$

Remark 4.2. Because $\lim _{\gamma \rightarrow \infty} \gamma /\left(c \rho_{\gamma+\delta}\right)=1$ (see Albrecher et al. (2012, Example 2.1)), taking limits in (4.2) leads us to

$$
\begin{aligned}
\lim _{\gamma \rightarrow \infty} V_{L}(u ; b) & =\frac{1}{\beta} \frac{\left(\beta-\alpha_{1}\right)\left(\beta-\alpha_{2}\right)\left(e^{\alpha_{1} u}-e^{\alpha_{2} u}\right)}{\left(\beta-\alpha_{2}\right) \alpha_{1} e^{\alpha_{1} b}-\left(\beta-\alpha_{1}\right) \alpha_{2} e^{\alpha_{2} b}}=\frac{1}{\beta} \frac{e^{\alpha_{1} u}-e^{\alpha_{2} u}}{\frac{\alpha_{1}}{\beta-\alpha_{1}} e^{\alpha_{1} b}-\frac{\alpha_{2}}{\beta-\alpha_{2}} e^{\alpha_{2} b}} \\
& =\frac{\lambda}{\beta} \frac{e^{\alpha_{1} u}-e^{\alpha_{2} u}}{\left(c \alpha_{1}+\delta\right) e^{\alpha_{1} b}-\left(c \alpha_{2}+\delta\right) e^{\alpha_{2} b}}, \quad 0 \leq u \leq b,
\end{aligned}
$$

where the last equality follows from the fact that $\alpha_{1}$ and $\alpha_{2}$ both satisfy (4.1). As expected, the final expression is identical to equation (3.5) in Avanzi et al. (2007), who studied the dual model with the classical barrier.

\subsection{Optimal dividend barrier}

Note that (4.2) factorizes $V_{L}(u ; b)$ as the product of a function of $u$ and a function of $b$. This implies that the optimal $b^{*}$ (if positive) which maximizes $V_{L}(u ; b)$ with respect to $b$ is independent of the initial capital $u$, as long as $0 \leq u \leq b^{*}$. By setting $\partial V_{L}(u ; b) / \partial b=0$, we arrive at

$$
b^{*}=\frac{1}{\alpha_{1}-\alpha_{2}} \ln \frac{\left[\beta \delta\left(\rho_{\gamma+\delta}+\alpha_{2}\right)+\gamma \alpha_{2}\left(\beta+\rho_{\gamma+\delta}\right)\right]\left(\beta-\alpha_{1}\right) \alpha_{2}}{\left[\beta \delta\left(\rho_{\gamma+\delta}+\alpha_{1}\right)+\gamma \alpha_{1}\left(\beta+\rho_{\gamma+\delta}\right)\right]\left(\beta-\alpha_{2}\right) \alpha_{1}} .
$$

After some tedious algebra, it is found that the same $b^{*}$ satisfies

$$
\left.\frac{\partial}{\partial b} V_{U}(u ; b)\right|_{b=b^{*}}=0, \quad u \geq b^{*} .
$$

In other words, it is actually the same $b^{*}$ that maximizes $V(u ; b)$ with respect to $b$ for any initial surplus $u \geq 0$. Interestingly, it can also be shown that

$$
V_{L}^{\prime}\left(b^{*}-; b^{*}\right)=V_{U}^{\prime}\left(b^{*}+; b^{*}\right)=1 .
$$

While the smooth pasting condition is already known from (3.9), at $u=b^{*}$ both the derivatives are equal to 1 . We refer to interested readers to Albrecher et al. (2011b, Section 8, Remark (ii)) regarding the interpretation of $b^{*}$ as the optimal financial capital. 
Moreover, by considering

one concludes that

$$
\left.\frac{d}{d b} V(b ; b)\right|_{b=b^{*}}=\left.\frac{\partial}{\partial u} V(u ; b)\right|_{u=b=b^{*}}+\left.\frac{\partial}{\partial b} V(u ; b)\right|_{u=b=b^{*}}=V^{\prime}\left(b^{*} ; b^{*}\right)+0=1,
$$

$$
\left.\frac{\partial}{\partial b}[u-b+V(b ; b)]\right|_{b=b^{*}}=-1+\left.\frac{d}{d b} V(b ; b)\right|_{b=b^{*}}=0,
$$

which shows that it is still the same $b^{*}$ that maximizes the expected discounted dividends until ruin even if time 0 is declared to be a dividend decision time.

\section{Numerical studies}

In this section, we illustrate the results of this paper, as well as the impact of its assumptions about the payment of dividends and the occurrence of ruin. To that purpose we will consider the following three models:

Model CC: The dual model with the classical dividend barrier, whereby both dividend payments and ruin are monitored continuously.

For this model, the expected present value of dividends is computed according to Avanzi et al. (2007), whereas the Laplace transform of the time to ruin according to Cheung and Drekic (2008). Note that this dividend strategy is known to be optimal for at least exponential and mixture of exponential gains (see e.g. Avanzi et al., 2011).

Model PP: The dual model whereby both dividend payments and ruin can only occur at the same (periodic) Erlang distributed random intervals.

Assumptions about dividends and ruin are here identical to that of Albrecher et al. (2011a), and ruin is assumed not to be observed at time 0 . That is, we allow zero initial surplus at time 0 without ruin occurring. Their results about the expected present value of dividends can readily be adapted to the dual model by reversing the roles of $g_{\delta,+}(\cdot)$ and $g_{\delta,-}(\cdot)$ in their equation $(37)^{1}$; this is done in Appendix A for exponentially distributed inter-dividend-decision intervals and gains and for $0 \leq u \leq b$.

Model PC: The dual model whereby ruin is allowed to occur at any time but dividends are allowed to be paid only at Erlang distributed random intervals.

This is the model that is developed in this paper, and both the expected present value of dividends and the Laplace transform of the time to ruin are calculated according to Sections 3 and 2, respectively.

Throughout, we will consider four gains distributions with rational Laplace transform, namely

$$
\begin{array}{ll}
\text { Mixture of exponentials: } & p(y)=(1 / 3) 2 e^{-2 y}+(2 / 3) 0.8 e^{-0.8 y} ; \\
\text { Exponential: } & p(y)=e^{-y} ; \\
\text { Combination of exponentials: } & p(y)=(2) 1.5 e^{-1.5 y}+(-1) 3 e^{-3 y} ; \\
\text { Erlang(2): } & p(y)=4 y e^{-2 y}
\end{array}
$$

These four distributions have a mean of 1 and a decreasing variance; they are identical to the ones considered in Avanzi et al. (2007). We always set $\delta=0.01$. Unless stated otherwise, we will use $\lambda=1$ and $c=0.75$ such that the expected profit per time unit (in the absence of dividends) is $1-c=0.25$. For example, under these default parameters the values of $b_{C C}^{*}$ (the optimal barrier of Model CC) for the four gains distributions are $11.05822,10.61754,9.57551$ and 9.45414 respectively.

In the next subsection we will illustrate the convergence of the variables of interest in Models PP and PC to that of Model CC, when the expected inter-dividend-decision time interval tends to 0. In Section 5.2 we discuss the convergence of Model PC when the variance of the inter-dividend-decision times tends to 0 . Finally, Section 5.3 is concerned with the level of the barrier that maximizes the expected present value of dividends in Model PC, and its convergence to the optimal barrier of Model CC.

${ }^{1}$ Note that the quantities $g_{\delta,+}(\cdot)$ and $g_{\delta,-}(\cdot)$ in Albrecher et al. (2011a) have the same definition as in (3.15). 


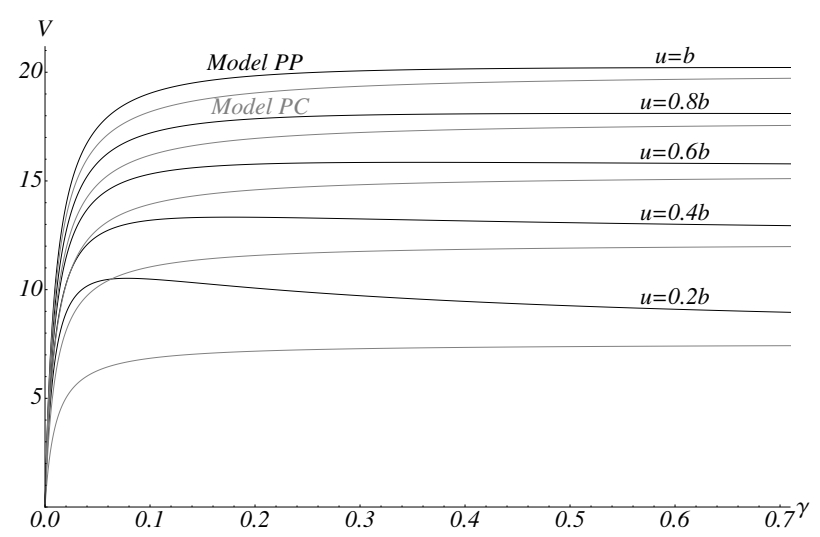

(a) $c=0.8, \mu=1 / \lambda=1, b=b_{C C}^{*}=10.70$

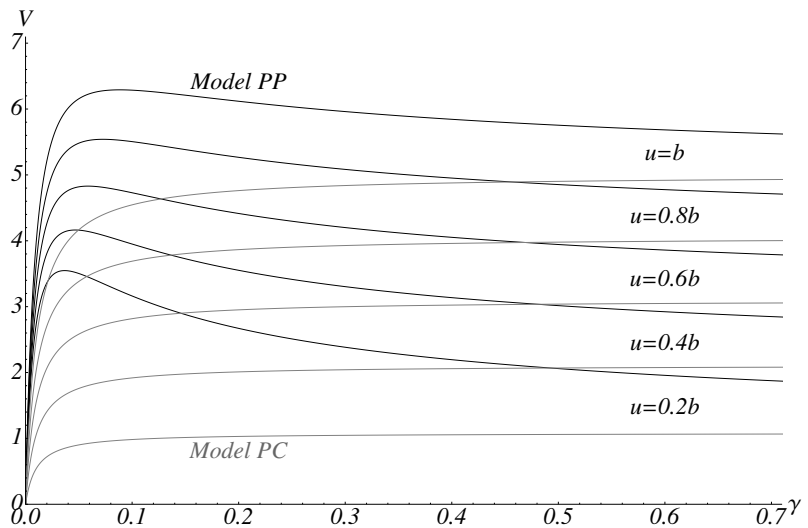

(b) $c=0.95, \mu=1 / \lambda=1, b=b_{C C}^{*}=4.675$

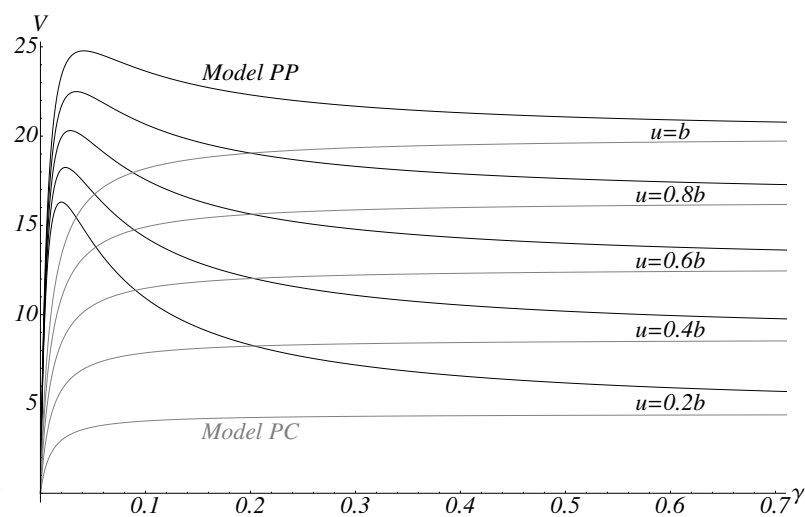

(c) $c=0.8, \mu=1 / \lambda=50, b=b_{C C}^{*}=17.59$

Figure 2: Expected present value of dividends in Models PP and PC with exponential claims using the optimal barrier $b_{C C}^{*}$ (according to Model CC). The curve for Model PP shows a more pronounced hump when risk increases because of smaller expected profit ((a) to (b)) and/or because of more variable gains ((a) to (c)).

\subsection{The impact of the expected inter-dividend-decision times}

In this subsection, we consider exponential inter-dividend-decision times (i.e. $n=1$ ) and vary $\gamma$ in order to illustrate the impact of the expected inter-dividend-decision time interval $1 / \gamma$.

Figure 2 illustrates the impact of decreasing expected inter-dividend-decision times (i.e. increasing $\gamma$ 's) on the expected present value of dividends, in three different configurations of parameters. Recall that under the assumptions that solvency is monitored continuously and gains are (mixed) exponential, a continuously monitored barrier strategy at the corresponding optimal barrier level $b_{C C}^{*}$ is the globally optimal dividend strategy (see, for instance, Avanzi et al., 2011). Hence, the expected present value of dividends under Model $\mathrm{PC}$ at barrier level $b_{C C}^{*}$ will necessarily be inferior to that of Model CC. Furthermore, observe that the impact of $\gamma$ in Model PC is such that higher $\gamma$ will lead to more dividends, as these can be paid more often. The expected present value of dividends is thus monotonically increasing in $\gamma$ towards the corresponding value under Model CC.

When ruin cannot occur between dividend payment times as in Model PP, a trade-off appears: as the expected inter-dividend-decision time interval decreases, more dividends can be paid, but the event of is also monitored more frequently. Because of this trade-off, the expected present value of dividends is sometimes first increasing and then decreasing in $\gamma$. This is more so for higher levels of risk, where the hump becomes more pronounced. By higher levels of risk, we mean either: (1) lower levels of initial surplus (as shown in all 


\begin{tabular}{c|c|ccccc} 
Model & $\gamma$ & $u=0$ & $u=0.1 b$ & $u=0.2 b$ & $u=0.5 b$ & $u=b$ \\
\hline PC & 1 & 0 & 6.17494 & 10.60689 & 18.28124 & 24.75248 \\
PC & 10 & 0 & 6.23046 & 10.70226 & 18.44560 & 24.97502 \\
PC & 1000 & 0 & 6.23662 & 10.71285 & 18.46386 & 24.99975 \\
PC & 90000 & 0 & 6.23668 & 10.71296 & 18.46405 & 25.00000 \\
CC & $(\infty)$ & 0 & 6.23668 & 10.71300 & 18.46400 & 25.00000
\end{tabular}

Table 1: Expected present value of dividends with mixture of exponentials gains

\begin{tabular}{c|c|ccccc} 
Model & $\gamma$ & $u=0$ & $u=0.1 b$ & $u=0.2 b$ & $u=0.5 b$ & $u=b$ \\
\hline PC & 1 & 0 & 6.41275 & 10.93153 & 18.53165 & 24.75247 \\
PC & 10 & 0 & 6.47040 & 11.02981 & 18.69826 & 24.97502 \\
PC & 1000 & 0 & 6.47681 & 11.04073 & 18.71678 & 24.99974 \\
PC & 90000 & 0 & 6.47687 & 11.04084 & 18.71696 & 24.99999 \\
CC & $(\infty)$ & 0 & 6.47688 & 11.04085 & 18.71697 & 25.00000 \\
PP & 90000 & 0.00006 & 6.47692 & 11.04087 & 18.71697 & 25.00000 \\
PP & 1000 & 0.00545 & 6.48061 & 11.04345 & 18.71798 & 25.00049 \\
PP & 10 & 0.49783 & 6.81747 & 11.27692 & 18.80751 & 25.04190 \\
PP & 1 & 3.16064 & 8.60229 & 12.47657 & 19.18494 & 25.12328
\end{tabular}

Table 2: Expected present value of dividends with exponential gains

\begin{tabular}{c|c|ccccc} 
Model & $\gamma$ & $u=0$ & $u=0.1 b$ & $u=0.2 b$ & $u=0.5 b$ & $u=b$ \\
\hline PC & 1 & 0 & 6.99467 & 11.71399 & 19.11949 & 24.75247 \\
PC & 10 & 0 & 7.05755 & 11.81931 & 19.29139 & 24.97502 \\
PC & 1000 & 0 & 7.06454 & 11.83101 & 19.31049 & 24.99975 \\
PC & 90000 & 0 & 7.06461 & 11.83113 & 19.31068 & 24.99999 \\
CC & $(\infty)$ & 0 & 7.06461 & 11.83113 & 19.31068 & 25.00000
\end{tabular}

Table 3: Expected present value of dividends with combination of exponential gains

\begin{tabular}{c|c|ccccc} 
Model & $\gamma$ & $u=0$ & $u=0.1 b$ & $u=0.2 b$ & $u=0.5 b$ & $u=b$ \\
\hline PC & 1 & 0 & 7.07126 & 11.81478 & 19.19108 & 24.75247 \\
PC & 10 & 0 & 7.13484 & 11.92102 & 19.36363 & 24.97502 \\
PC & 1000 & 0 & 7.14190 & 11.93281 & 19.38280 & 24.99974 \\
PC & 90000 & 0 & 7.14197 & 11.93281 & 19.38299 & 24.99999 \\
CC & $(\infty)$ & 0 & 7.14198 & 11.93294 & 19.38300 & 25.00000
\end{tabular}

Table 4: Expected present value of dividends with Erlang(2) gains

three graphs); (2) lower expected profit per unit time $\lambda \mu-c$ (compare graph (a) with graph (b), where the expected profit goes from 0.2 to 0.05 ); or (3) an exponential gain distribution with higher variance (compare graph (a) with graph (c), where the coefficient of variation of gains goes from 1 to 50 with fixed expected profit). Note also that values for Model PP in Figure 2 are all strictly above that of Model PC. This makes sense, as ruin occurs earlier in Model PC, everything else being equal.

When the expected inter-dividend-decision time tends to zero, the expected present value of dividends and the Laplace transform of the time to ruin in both Models PP and PC approach that of Model CC, which was to be expected. Some of the results are illustrated in Tables 1-4 for all four gains distributions when $b=b_{C C}^{*}$, albeit only for the expected present value of dividends (for sake of brevity).

\subsection{Convergence of Model PC when the variance of inter-dividend-decision times tends to 0}

In this subsection, we focus on Model PC and set the expected inter-dividend-decision time interval $n / \gamma$ to 10 . As $n$ increases, the variance of the interval decreases towards 0 . 


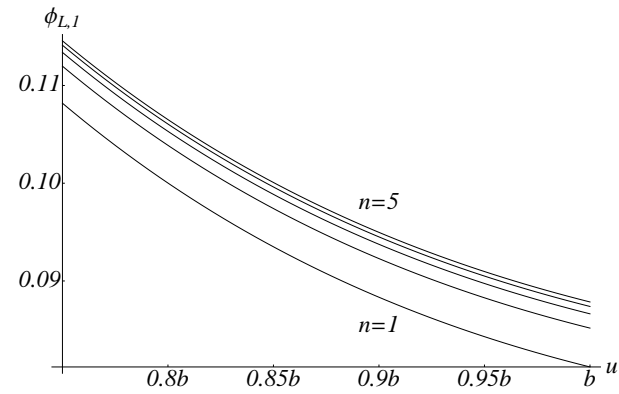

(a) Mixture of exponential gains

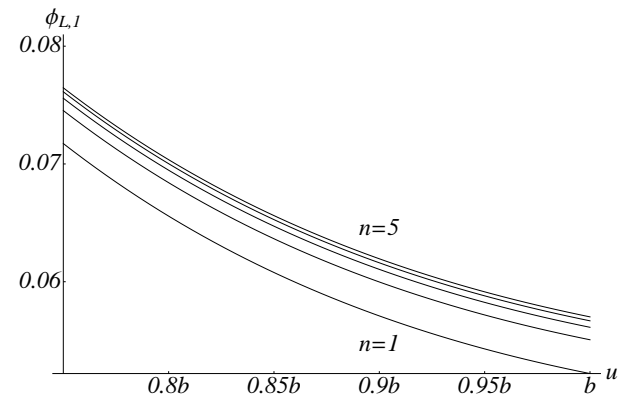

(c) Combination of exponential gains

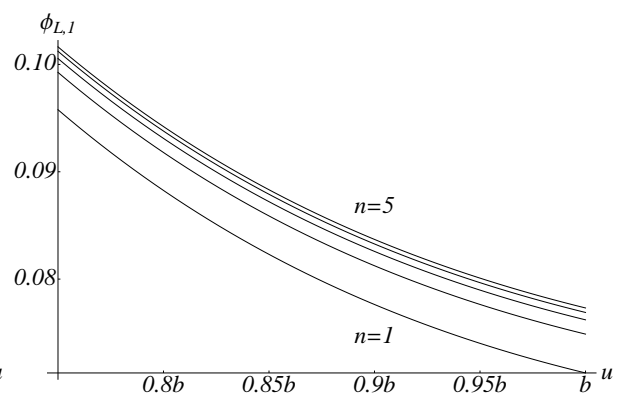

(b) Exponential gains

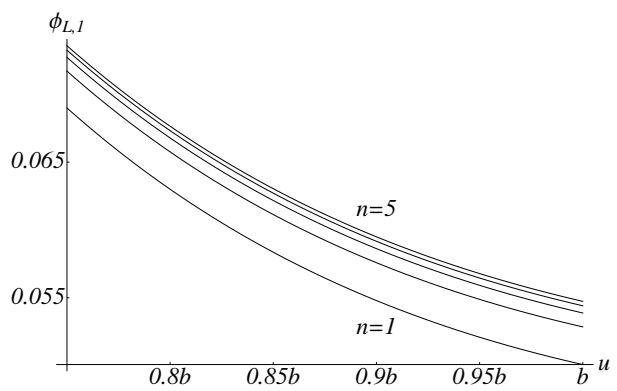

(d) Erlang(2) gains

Figure 3: Convergence of the Laplace transform of the time to ruin in Model PC when $n$ increases from 1 to 5

When we increase $n$ further, some numerical issues can arise when using standard mathematical software such as Mathematica, Matlab or Maple. Firstly, (2.30) and (3.28) require the inversion of an $n \times n$ matrix, which leads to a solution that is in the form of a ratio of two rational functions of high degree. This issue can be alleviated by determining the poles and residuals of that fraction in order to write the inverse of the Laplace transform explicitly, which simplifies its inversion and improves accuracy. Secondly, once the forms of $\phi_{L, i}(u ; b)$ and $V_{L, i}(u ; b)(i=1,2, \ldots, n)$ are obtained, the boundary conditions are used to determine the constant terms $\phi_{L, i}(b ; b)$ and $V_{L, i}(b ; b)(i=1,2, \ldots, n)$; see the procedure described at the end of Sections 2 and 3 . This involves solving a high dimensional system, with coefficients which turn out to be comparatively large. These create some numerical instability for larger $n$.

For our model, the convergence of the Laplace transform of the time to ruin when $n$ increases up to 5 is illustrated in Figure 3 when $b=b_{C C}^{*}$ (note that both the vertical and horizontal axes have been magnified). Similar behaviour is observed for expected present value of dividends. The convergence we observed is similar to that observed in e.g. Asmussen et al. (2002), Stanford et al. (2005), Ramaswami et al. (2008) or Albrecher et al. (2011a).

\subsection{The optimal barrier level $b_{P C}^{*}$ in Model $P C$}

What is the impact on the optimal barrier level of the periodicity of dividend payments and the variability of gains? To provide some answers we have determined numerically the optimal barrier level $b_{P C}^{*}$ (previously denoted by $b^{*}$ in Section 4.3) in Model PC for varying levels of $\gamma$ as well as different gain distributions. We assume here exponential inter-dividend-decision time intervals with mean $1 / \gamma$.

Table 5 shows that as $\gamma$ increases (i.e. the expected inter-dividend-decision times become shorter), the optimal barrier $b_{P C}^{*}$ under Model PC approaches that of Model CC from below. Furthermore, for each fixed $\gamma, b_{P C}^{*}$ appears to increase with the variance of the gains, which is likely due to the additional safety required for increased risk.

Figure 4 displays the expected present value of dividends until ruin in Models $\mathrm{CC}$ and PC as functions of the initial surplus (on the left) and dividend barrier (on the right). Whilst Model CC dominates Model PC 


\begin{tabular}{c|cccc}
$\gamma$ & Mixture & Exponential & Combination & Erlang $(2)$ \\
\hline 0.5 & 10.27032 & 9.84234 & 8.84003 & 8.72157 \\
1 & 10.57561 & 10.13961 & 9.11456 & 8.99413 \\
5 & 10.92803 & 10.48747 & 9.44627 & 9.32487 \\
10 & 10.98888 & 10.54820 & 9.50627 & 9.38491 \\
1000 & 11.05747 & 10.61651 & 9.57477 & 9.45340 \\
$(\infty)$ & 11.05821 & 10.61754 & 9.57551 & 9.45414
\end{tabular}

Table 5: Convergence of the (numerically) optimal barrier level $b_{P C}^{*}$ in Model PC to the optimal $b_{C C}^{*}$ of Model CC.

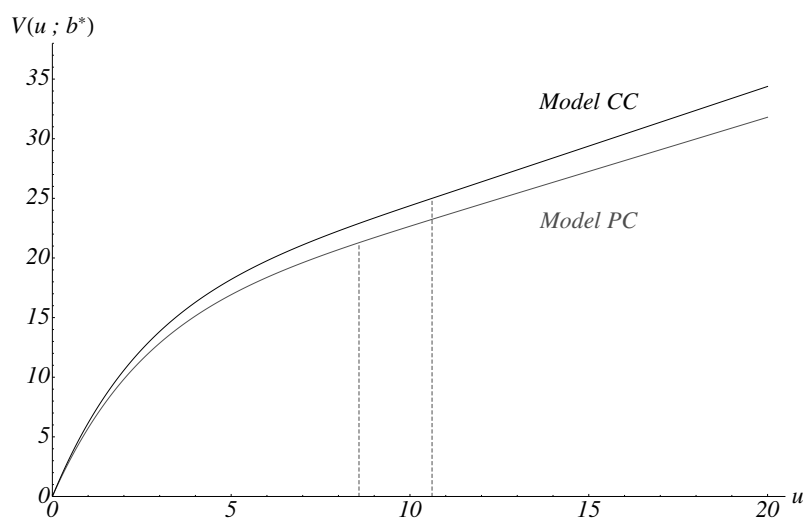

(a) $\gamma=0.1, n=1$

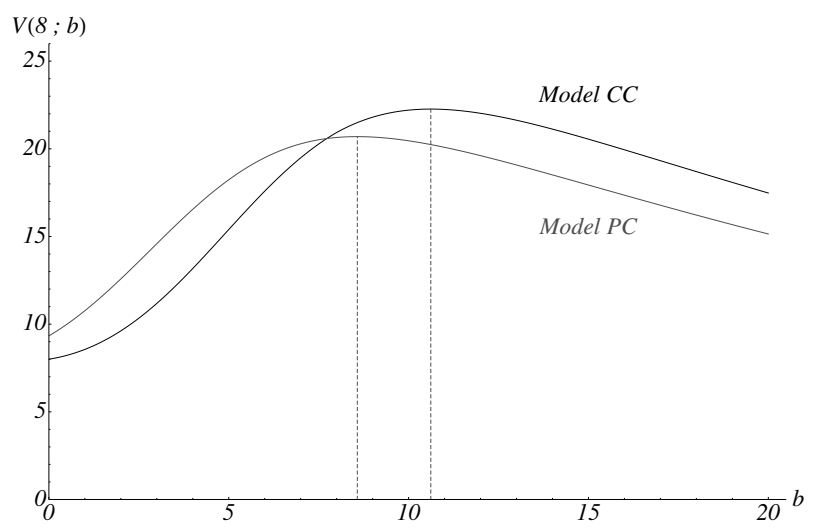

(b) $\gamma=0.1, n=1$

Figure 4: Illustration of the expected present value of dividends in Models CC and PC when gains are exponential, as a function of the initial surplus on the left, and as a function of the barrier level on the right. The vertical dashed lines show the associated optimal barrier levels 10.62 for Model CC and 8.57 for Model PC.

when the respective optimal barriers are applied for all $u$ (as it should, at least for mixed exponential gains), if one fixes for example $u=8$ then Model PC fares better when barrier levels are low (well below optimal). This is likely to be because of the additional safety related to delayed dividend payments, when the barrier is less than optimal. Conversely, the time value of money clearly does not justify delaying dividend payments any further when barrier levels are too high (above optimal).

Remark 5.1. Recall the discussion in Section 4.3 of the optimal barrier in Model PC when both interdividend-decision times and gains are exponential. Do numerical calculations suggest that some of the properties found therein hold in general?

First, it seems that the value of the optimal barrier in Model PC does not depend on the initial surplus. We were unable to find a counterexample numerically, when using all four distributions considered in this section, as well as varying $n$ from 1 to 5 .

Furthermore, $V_{L, 1}^{\prime}\left(b_{P C}^{*}-; b_{P C}^{*}\right)$ and $V_{U, 1}^{\prime}\left(b_{P C}^{*}+; b_{P C}^{*}\right)$ are numerically both equal to one for the whole (wide) range of parameters and gains distributions we tested. This suggests that the optimal dividend barrier $b_{P C}^{*}$ in this setting could be found by setting additional boundary conditions accordingly.

\section{Acknowledgements}

The authors would like to thank an anonymous referee for helpful comments and suggestions. Benjamin Avanzi and Bernard Wong would like to acknowledge support from an Australian School of Business Research Grant. Support from the Research Grants Council of the Hong Kong Special Administrative Region (Project Number: 701212) is also gratefully acknowledged by Eric Cheung, as is support for J.-K. Woo from a startup fund provided by the Faculty of Science and the Department of Statistics and Actuarial Science at the University of Hong Kong. 
Parts of this work were completed when Eric Cheung and J.-K. Woo visited the School of Risk and Actuarial Studies at the University of New South Wales, and when Benjamin Avanzi and Bernard Wong visited the Department of Statistics and Actuarial Science at the University of Hong Kong. The host institutions' hospitality during these visits are greatly appreciated.

\section{A. Dividends in the dual model when ruin can occur only at dividend payment times}

In this Appendix, we replicate the results of Albrecher et al. (2011a, Section 3.1) for exponential interdividend-decision times $Z_{k}$ 's and exponential gains with mean $1 / \gamma$ and $1 / \beta$ respectively, albeit in the dual model. The function $g_{\delta}(y)$ defined via (3.14) and (3.15) is still applicable, but the roles of $g_{\delta,+}(\cdot)$ and $g_{\delta,-}(\cdot)$ need to be interchanged when we apply equation (28) of Albrecher et al. (2011a) which now becomes

$$
\begin{aligned}
V(u ; b)= & \int_{b-u}^{\infty} \frac{\gamma\left(\beta-R_{\gamma+\delta}\right)}{c\left(\rho_{\gamma+\delta}+R_{\gamma+\delta}\right)} e^{-R_{\gamma+\delta} y}[y-(b-u)+V(b ; b)] d y+\int_{0}^{b-u} \frac{\gamma\left(\beta-R_{\gamma+\delta}\right)}{c\left(\rho_{\gamma+\delta}+R_{\gamma+\delta}\right)} e^{-R_{\gamma+\delta} y} V(u+y ; b) d y \\
& +\int_{0}^{u} \frac{\gamma\left(\beta+\rho_{\gamma+\delta}\right)}{c\left(\rho_{\gamma+\delta}+R_{\gamma+\delta}\right)} e^{-\rho_{\gamma+\delta} y} V(u-y ; b) d y, \quad 0 \leq u \leq b,
\end{aligned}
$$

where $-\rho_{\gamma+\delta}<0$ and $R_{\gamma+\delta}>0$ are the unique negative and positive roots of the quadratic equation (in $\xi$ )

$$
c \xi^{2}+(\lambda+\gamma+\delta-\beta c) \xi-(\gamma+\delta) \beta=0 .
$$

(The notation $\rho_{\gamma+\delta}$ is consistent with the one defined previously in Lemma 1. See also Remark 2.1.) Applying the operator $\left(d / d u+\rho_{\gamma+\delta}\right)\left(d / d u-R_{\gamma+\delta}\right)$ to the integral equation (A.1) and rearranging yields

$$
V^{\prime \prime}(u ; b)+\left(\rho_{\gamma+\delta}-R_{\gamma+\delta}-\frac{\gamma}{c}\right) V^{\prime}(u ; b)+\left(\frac{\gamma \beta}{c}-\rho_{\gamma+\delta} R_{\gamma+\delta}\right) V(u ; b)=0,
$$

which indicates that $V(u ; b)$ is of the form

$$
V(u ; b)=C_{1} e^{\alpha_{1} u}+C_{2} e^{\alpha_{2} u},
$$

where $\alpha_{1}$ and $\alpha_{2}$ (see Remark A.1) are the roots of the characteristic equation (in $\xi$ )

$$
\xi^{2}+\left(\rho_{\gamma+\delta}-R_{\gamma+\delta}-\frac{\gamma}{c}\right) \xi+\frac{\gamma \beta}{c}-\rho_{\gamma+\delta} R_{\gamma+\delta}=0
$$

and $C_{1}$ and $C_{2}$ are constants that do not depend on $u$ but possibly depend on $b$. The constants $C_{1}$ and $C_{2}$ are determined by back substituting (A.3) into (A.1). After some algebra, matching the coefficients of $e^{R_{\gamma+\delta} u}$ yields

$$
\frac{\alpha_{1} e^{\alpha_{1} b}}{R_{\gamma+\delta}-\alpha_{1}} C_{1}+\frac{\alpha_{2} e^{\alpha_{2} b}}{R_{\gamma+\delta}-\alpha_{2}} C_{2}=\frac{1}{R_{\gamma+\delta}}
$$

whereas the coefficients of $e^{-\rho_{\gamma+\delta} u}$ imply

$$
\frac{1}{\rho_{\gamma+\delta}+\alpha_{1}} C_{1}+\frac{1}{\rho_{\gamma+\delta}+\alpha_{2}} C_{2}=0 .
$$

Solving the above two equations for $C_{1}$ and $C_{2}$ and substituting into (A.3), we ultimately arrive at

$$
V(u ; b)=\frac{1}{R_{\gamma+\delta}} \frac{\left(R_{\gamma+\delta}-\alpha_{1}\right)\left(R_{\gamma+\delta}-\alpha_{2}\right)\left[\left(\rho_{\gamma+\delta}+\alpha_{1}\right) e^{\alpha_{1} u}-\left(\rho_{\gamma+\delta}+\alpha_{2}\right) e^{\alpha_{2} u}\right]}{\left(\rho_{\gamma+\delta}+\alpha_{1}\right)\left(R_{\gamma+\delta}-\alpha_{2}\right) \alpha_{1} e^{\alpha_{1} b}-\left(\rho_{\gamma+\delta}+\alpha_{2}\right)\left(R_{\gamma+\delta}-\alpha_{1}\right) \alpha_{2} e^{\alpha_{2} b}}, \quad 0 \leq u \leq b .
$$

Remark A.1. By applying Vieta's rule to the quadratic equation (A.2) one has

$$
\rho_{\gamma+\delta} R_{\gamma+\delta}=\frac{(\gamma+\delta) \beta}{c} \quad \text { and } \quad \rho_{\gamma+\delta}-R_{\gamma+\delta}=\frac{\lambda+\gamma+\delta}{c}-\beta,
$$

and therefore (A.4) can be rewritten as (4.1), which is in turn equivalent to (A.2) with $\gamma=0$. This means that one can set $\alpha_{1}=R_{\delta}$ and $\alpha_{2}=-\rho_{\delta}$ according to our notations. 
Remark A.2. From Albrecher et al. (2011a, Remark 2.1), it is known that $\rho_{\gamma+\delta} \rightarrow \infty$ and $R_{\gamma+\delta} \rightarrow \beta$ as $\gamma \rightarrow \infty$. Hence, taking limit in (A.5) again leads us to the first equality in (4.3) which is again expected, since the limit $\gamma \rightarrow \infty$ in this model also corresponds to the dual model under the classical barrier strategy. 


\section{References}

Albrecher, H., Badescu, A. L., Landriault, D., 2008. On the dual risk model with tax payments. Insurance: Mathematics and Economics 42 (3), 1086-1094.

Albrecher, H., Cheung, E. C. K., Thonhauser, S., 2011a. Randomized observation periods for the compound poisson risk model: Dividends. ASTIN Bulletin 41 (2), 645-672.

Albrecher, H., Cheung, E. C. K., Thonhauser, S., 2012. Randomized observation periods for the compound poisson risk model: The discounted penalty function. Scandinavian Actuarial Journal in press.

Albrecher, H., Gerber, H. U., Shiu, E. S. W., 2011b. The optimal dividend barrier in the gamma-omega model. European Actuarial Journal 1 (1), 43-55.

Asmussen, S., Avram, F., Usabel, M., 2002. Erlangian approximations for finite-horizon ruin probabilities. ASTIN Bulletin $32(2), 267-281$.

Avanzi, B., 2009. Strategies for dividend distribution: A review. North American Actuarial Journal 13 (2), $217-251$.

Avanzi, B., Gerber, H. U., 2008. Optimal dividends in the dual model with diffusion. ASTIN Bulletin 38 (2), $653-667$.

Avanzi, B., Gerber, H. U., Shiu, E. S. W., 2007. Optimal dividends in the dual model. Insurance: Mathematics and Economics 41 (1), 111-123.

Avanzi, B., Shen, J., Wong, B., 2011. Optimal dividends and capital injections in the dual model with diffusion. ASTIN Bulletin $41(2), 611-644$.

Bayraktar, E., Egami, M., 2008. Optimizing venture capital investments in a jump diffusion model. Mathematical Methods of Operations Research 67 (1), 21-42.

Bühlmann, H., 1970. Mathematical Methods in Risk Theory. Grundlehren der mathematischen Wissenschaften. Springer-Verlag, Berlin, Heidelberg, New York.

Cheung, E. C. K., 2008. Discussion on a. badescu and d. landriault's "recursive calculation of the dividend moments in a multi-threshold risk model". North American Actuarial Journal 12 (3), 336-340.

Cheung, E. C. K., 2010. Discussion on h. albrecher, h. u. gerber and h. yang's "a direct approach to the discounted penalty function". North American Actuarial Journal 14 (4), 441-445.

Cheung, E. C. K., 2011. On a class of stochastic models with two-sided jumps. Queueing Systems: Theory and Applications 69 (1), 1-28

Cheung, E. C. K., 2012. A unifying approach to the analysis of business with random gains. Scandinavian Actuarial Journal in press.

Cheung, E. C. K., Drekic, S., 2008. Dividend moments in the dual risk model: Exact and approximate approaches. ASTIN Bulletin 38 (2), 149-159.

Cramér, H., 1955. Collective Risk Theory: A Survey of the Theory from the Point of View of the Theory of Stochastic Processes. Ab Nordiska Bokhandeln, Stockholm.

de Finetti, B., 1957. Su un'impostazione alternativa della teoria collettiva del rischio. Transactions of the XVth International Congress of Actuaries 2, 433-443.

Gerber, H. U., Shiu, E. S. W., 1998. On the time value of ruin. North American Actuarial Journal 2 (1), $48-78$.

Gerber, H. U., Smith, N., 2008. Optimal dividends with incomplete information in the dual model. Insurance: Mathematics and Economics $43(2), 227-233$.

Grandell, J., 1991. Aspects of Risk Theory. Springer, New York.

Landriault, D., Renaud, J.-F., Zhou, X., 2012. Insurance risk models with parisian implementation delays, preprint.

Lundberg, F., 1909. Über die Theorie der Rückversicherung. Transactions of the VIth International Congress of Actuaries 1, $877-948$.

Mazza, C., Rullière, D., 2004. A link between wave governed random motions and ruin processes. Insurance: Mathematics and Economics 35 (2), 205-222.

Miyasawa, K., 1962. An economic survival game. Journal of the Operations Research Society of Japan 4 (3), 95-113.

$\mathrm{Ng}$, A. C. Y., 2009. On a dual model with a dividend threshold. Insurance: Mathematics and Economics 44 (2), 315-324.

$\mathrm{Ng}$, A. C. Y., 2010. On the upcrossing and downcrossing probabilities of a dual risk model with phase-type gains. ASTIN Bulletin 40 (1), 281-306

Ramaswami, V., Woolford, D. G., Stanford, D. A., 2008. The erlangization method for markovian fluid flows. Annals of Operations Research 160 (1), 215-225.

Seal, H. L., 1969. Stochastic Theory of a Risk Business. Wiley Series in Probability and Mathematical Statistics-Applied. John Wiley \& Sons, Inc., New York, London, Sydney, Toronto.

Stanford, D. A., Avram, F., Badescu, A. L., Breuer, L., Da Silva Soares, A., Latouche, G., 2005. Phase-type approximations to finite-time ruin probabilities in the sparre-andersen and stationary renewal risk models. ASTIN Bulletin 35 (1), $131-144$.

Stanford, D. A., Yu, K., Ren, J., 2011. Erlangian approximation to finite time ruin probabilities in perturbed risk models. Scandinavian Actuarial Journal 2011 (1), 38-58.

Takács, L., 1967. Combinatorial Methods in the Theory of Stochastic Processes. John Wiley \& Sons, Inc., New York.

Takeuchi, K., 1962. A remark on economic survival game. Journal of the Operations Research Society of Japan 4 (3), $114-121$.

Zhu, J., Yang, H., 2008. Ruin probabilities of a dual markov-modulated risk model. Communications in Statistics - Theory and Methods 37 (20), 3298-3307. 